\title{
NEW RESULTS ON COMPLETE ELLIPTIC EQUATIONS WITH ROBIN BOUNDARY COEFFICIENT-OPERATOR CONDITIONS IN NON COMMUTATIVE CASE
}

M. Cheggag, Ecole Nationale Polytechnique d'Oran, Oran, Algérie, mustapha.cheggag@enp-oran.dz,

A. Favini, Dipartimento di Matematica, Università degli Studi di Bologna, Bologna, Italia, favini@dm.unibo.it,

R. Labbas, Normandie Univ, Le Havre, France, rabah.labbas@univ-lehavre.fr,

S. Maingot, Normandie Univ, Le Havre, France, stephane.maingot@univ-lehavre.fr,

Kh. Ould Melha, Laboratoire de Mathématiques Pures et Appliquées, Université Abdelhamid Ibn Badis, Mostaganem, Algérie, ould_melha_khel@yahoo.fr

In this paper, we prove some new results on operational second order differential equations of elliptic type with general Robin boundary conditions in a non-commutative framework. The study is performed when the second member belongs to a Sobolev space. Existence, uniqueness and optimal regularity of the classical solution are proved using interpolation theory and results on the class of operators with bounded imaginary powers. We also give an example to which our theory applies. This paper improves naturally the ones studied in the commutative case by M. Cheggag, A. Favini, R. Labbas, S. Maingot and A. Medeghri: in fact, introducing some operational commutator, we generalize the representation formula of the solution given in the commutative case and prove that this representation has the desired regularity.

Keywords: second-order elliptic differential equations; Robin boundary conditions in non commutative cases; analytic semigroup; maximal regularity.

Dedicated to Professor Angelo Favini on his 70th birthday.

\section{Introduction and Hypotheses}

Let $A, B, H$ be closed linear operators in a complex Banach space $X, f \in L^{p}(0,1 ; X)$, $1<p<\infty$ and $d_{0}, u_{1} \in X$. A recent paper [1] studies the problem

$$
\left\{\begin{array}{l}
u^{\prime \prime}(x)+2 B u^{\prime}(x)+A u(x)=f(x), x \in(0,1), \\
u^{\prime}(0)-H u(0)=d_{0}, u(1)=u_{1},
\end{array}\right.
$$

under some commutativity assumptions between $A, B, H$.

The authors have written the above equation in the following form

$$
\left\{\begin{array}{l}
u^{\prime \prime}(x)+(L-M) u^{\prime}(x)-L M u(x)=f(x), x \in(0,1) \\
u^{\prime}(0)-H u(0)=d_{0}, u(1)=u_{1}
\end{array}\right.
$$

where $L=B-\sqrt{B^{2}-A}$ and $M=-B-\sqrt{B^{2}-A}$ under some appropriated hypotheses and assuming the commutativity $L M=M L$. 
In this paper, in order to solve a larger class of boundary value problems, we want to drop the hypothesis $L M=M L$. To this end, we consider some operators $L_{\omega}, M_{\omega}$ depending on a parameter $\omega \geqslant 0$ and study the second order operational differential equation

$$
u^{\prime \prime}(x)+\left(L_{\omega}-M_{\omega}\right) u^{\prime}(x)-\frac{1}{2}\left(L_{\omega} M_{\omega}+M_{\omega} L_{\omega}\right) u(x)=f(x), x \in(0,1),
$$

together with the abstract boundary conditions of Robin's type

$$
\left\{\begin{array}{l}
u^{\prime}(0)-H u(0)=d_{0} \\
u(1)=u_{1} .
\end{array}\right.
$$

The idea to consider (3) is explained in $[2,3]$. Here $L_{\omega}, M_{\omega}$ are two closed linear operators in $X$ depending on parameter $\omega$.

We seek for a classical solution to (3), (4), that is a function $u$ such that

$$
\left\{\begin{array}{l}
u \in W^{2, p}(0,1 ; X), \\
x \mapsto\left(L_{\omega} M_{\omega}\right) u(x), x \mapsto\left(M_{\omega} L_{\omega}\right) u(x) \in L^{p}(0,1 ; X), \\
x \mapsto\left(L_{\omega}-M_{\omega}\right) u^{\prime}(x) \in L^{p}(0,1 ; X), \\
u(0) \in D(H), \\
u \text { satisfies (3) and (4). }
\end{array}\right.
$$

Generally, more regularity is required for $f$ to obtain a classical solution, unless $X$ has some particular geometrical properties. This is why we assume in all this study that

$$
X \text { is a } U M D \text { space. }
$$

Our hypotheses with respect to operators $H, L_{\omega}$ and $M_{\omega}$ are the following:

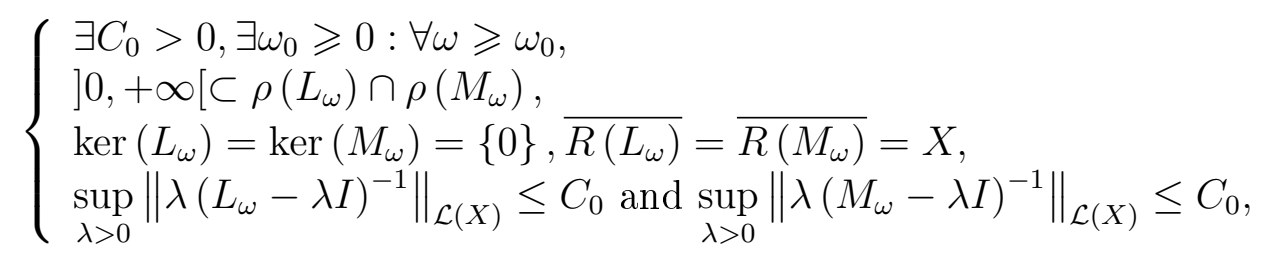

(here $\rho(P)$, ker $(P), R(P)$ denote respectively the resolvent set, the kernel and the range of operator $P$ ) and

$$
\left\{\begin{array}{l}
\left.\exists \theta_{1}, \theta_{2} \in\right] 0, \frac{\pi}{2}\left[, \exists C \geqslant 1: \forall \omega \geqslant \omega_{0}, \forall s \in \mathbb{R}\right. \\
\left(-L_{\omega}\right)^{i s},\left(-M_{\omega}\right)^{i s} \in \mathcal{L}(X), \\
\left\|\left(-L_{\omega}\right)^{i s}\right\|_{\mathcal{L}(X)} \leqslant C e^{\theta_{1}|s|} \text { and }\left\|\left(-M_{\omega}\right)^{i s}\right\|_{\mathcal{L}(X)} \leqslant C e^{\theta_{2}|s|}
\end{array}\right.
$$

Assumptions (7) and (8) involve that, for $\omega \geqslant \omega_{0},-L_{\omega}$ and $-M_{\omega}$ belong to the class $\operatorname{BIP}(X, \theta)$ [4, Definition 1, p. 431]. Since $\left.\theta_{1}, \theta_{2} \in\right] 0, \frac{\pi}{2}\left[, L_{\omega}\right.$ and $M_{\omega}$ generate bounded analytic semigroups in $X,\left(e^{\xi L_{\omega}}\right)_{\xi \geqslant 0},\left(e^{\xi M_{\omega}}\right)_{\xi \geqslant 0}[4$, p. 437].

For any $\omega \geqslant \omega_{0}$, we assume also

$$
\begin{gathered}
D\left(L_{\omega}\right)=D\left(M_{\omega}\right), \\
D\left(\left(L_{\omega}+M_{\omega}\right)^{2}\right) \subset D\left(\left(M_{\omega}-L_{\omega}\right)^{2}\right),
\end{gathered}
$$




$$
0 \in \rho\left(L_{\omega}+M_{\omega}\right)
$$

Set

$$
\Lambda_{\omega}:=\left(M_{\omega}-H\right)+e^{L_{\omega}} e^{M_{\omega}}\left(L_{\omega}+H\right),
$$

(which will be, in some sense, the abstract determinant of our problem), then we suppose that

$$
\forall \omega \geqslant \omega_{0}, 0 \in \rho\left(\Lambda_{\omega}\right)
$$

and

$$
\forall \xi \in D\left(L_{\omega}\right), \forall \omega \geqslant \omega_{0}, \Lambda_{\omega}^{-1} \xi \in D\left(\left(L_{\omega}+M_{\omega}\right)^{2}\right)
$$

Set

$$
C_{L_{\omega}, M_{\omega}}=\left(M_{\omega} L_{\omega}-L_{\omega} M_{\omega}\right)\left(L_{\omega}+M_{\omega}\right)^{-2}=\left[M_{\omega} ; L_{\omega}\right]\left(L_{\omega}+M_{\omega}\right)^{-2},
$$

then we will see that, under our hypotheses, $C_{L_{\omega}, M_{\omega}} \in \mathcal{L}(X)$. We assume in all this work, the following non-commutativity hypothesis

$$
\forall \omega \geqslant \omega_{0},\left\|C_{L_{\omega}, M_{\omega}}\right\|_{\mathcal{L}(X)} \leqslant \chi(\omega)
$$

where

$$
\chi:\left[\omega_{0},+\infty\left[\rightarrow \mathbb{R}^{+} \text {is such that } \lim _{\omega \rightarrow+\infty} \chi(\omega)=0 .\right.\right.
$$

In many concrete cases, the function $\chi$ has the following form

$$
\chi(\omega)=\frac{C}{\omega^{\alpha}} \text { for } \omega \text { large enough, with } C, \alpha>0 .
$$

The commutator defined in (15) was used for the first time by Favini et al. [2].

Remark 1. From assumption (11), we deduce that $\left(L_{\omega}+M_{\omega}\right)^{2}$ is closed.

Remark 2. We have not assumed that $L_{\omega}$ and $M_{\omega}$ are boundedly invertible as in Cheggag et al. $[1,5]$.

Remark 3. Assume (6) $\backsim(16)$. We then will see in Lemma 1 below, that

$$
D\left(L_{\omega} M_{\omega}\right) \cap D\left(M_{\omega} L_{\omega}\right)=D\left(\left(L_{\omega}+M_{\omega}\right)^{2}\right) .
$$

Suppose that Problem (3), (4) has a classical solution $u$. Then, from above

$$
u \in W^{2, p}(0,1 ; X) \cap L^{p}\left(0,1 ; D\left(\left(L_{\omega}+M_{\omega}\right)^{2}\right)\right),
$$

which implies

$$
u(0), u(1) \in\left(D\left(\left(L_{\omega}+M_{\omega}\right)^{2}\right), X\right)_{\frac{1}{2 p}, p}=\left(X, D\left(\left(L_{\omega}+M_{\omega}\right)^{2}\right)\right)_{1-\frac{1}{2 p}, p}
$$

(see [6]).

Remark 4. By the well known reiteration theorem, we have

$$
\begin{aligned}
\left(X, D\left(\left(L_{\omega}+M_{\omega}\right)^{2}\right)\right)_{1-\frac{1}{2 p}, p} & =\left(X, D\left(L_{\omega}+M_{\omega}\right)\right)_{2-\frac{1}{p}, p}=\left(X, D\left(L_{\omega}\right)\right)_{1+1-\frac{1}{p}, p} \\
& =\left\{\phi \in D\left(L_{\omega}\right): L_{\omega} \phi \in\left(X, D\left(L_{\omega}\right)\right)_{1-\frac{1}{p}, p}\right\},
\end{aligned}
$$


and in particular

$$
u(0), u(1) \in D\left(L_{\omega}\right)=D\left(M_{\omega}\right)
$$

The main result in this work is

Theorem 1. Assume (6) $\sim(16)$. Let $f \in L^{p}(0,1 ; X)$ with $1<p<\infty$. Then, there exists $\omega^{*} \geqslant \omega_{0}$ such that, for all $\omega \geqslant \omega^{*}$, the two following assertions are equivalent.

1. Problem (3), (4) has a unique classical solution.

$$
u_{1}, \Lambda_{\omega}^{-1} d_{0} \in\left(X, D\left(\left(L_{\omega}+M_{\omega}\right)^{2}\right)\right)_{1-\frac{1}{2 p}, p} .
$$

The plan of this paper is as follows. Section 1 contains some technical lemmas. In Section 2, we use the representation formula of the solution given in the commutative case and some heuristic consideration to obtain an integral equation verified by the eventual classical solution $u=\left(L_{\omega}+M_{\omega}\right)^{-2} v$ of Problem (3), (4). This integral equation is written in the form $v+R_{\omega}(v)=F_{\omega}(f)+\Gamma_{\omega}$, where $R_{\omega}, F_{\omega}$ and $\Gamma_{\omega}$ depend on $L_{\omega}$ and $M_{\omega}$. Section 3 contains the study of $F_{\omega}(f)+\Gamma_{\omega}$ and $R_{\omega}$, which will allow us to write

$$
u=\left(L_{\omega}+M_{\omega}\right)^{-2}\left(\left(I+R_{\omega}\right)^{-1}\left(F_{\omega}(f)+\Gamma_{\omega}\right)\right),
$$

for a large $\omega$. In section 4 , we will prove Theorem 1 by studying the regularity of the representation (21). Section 5 is devoted to some comparison with a recent paper [1]. In fact, we obtain, in the case when $L_{\omega} M_{\omega}=M_{\omega} L_{\omega}$, a result concerning Problem (2) which generalizes the one given in [1] (see Corollary 1). Finally, in Section 6, we give an application to which our results apply.

\section{Technical Lemmas}

In the following Lemmas, we do not need the dependence of $\omega$, so we drop $\omega$ in the notations and denote $L_{\omega}, M_{\omega}, \Lambda_{\omega}$ by $L, M, \Lambda$. We omit some proofs; some are obvious, others can be found in Favini et al. [2, Lemmas 2.1 and 2.2].

Lemma 1. Assume (7) (10). Then

1. $D(L M)=D\left(M^{2}\right)$ and $D(M L)=D\left(L^{2}\right)$.

2. $D\left((L+M)^{2}\right)=D\left(L^{2}\right) \cap D\left(M^{2}\right)=D(L M) \cap D(M L)$.

3. $D\left((L-M)^{2}-(L+M)^{2}\right)=D(L M) \cap D(M L)=D\left((L+M)^{2}\right)$.

1. See [2, Lemma 2.2, p. 1499].

2. The proof can be found in [7]. We give the details for the commodity of the readers. Let $\phi \in D\left((L+M)^{2}\right)$. There exists $\xi \in X$ such that

$$
\phi=(L+M)^{-2} \xi,
$$

so

$$
\begin{gathered}
M \phi=M(L+M)^{-2} \xi=\frac{1}{2}\left(I-(L+M-2 M)(L+M)^{-1}\right)(L+M)^{-1} \xi \\
=\frac{1}{2}\left(I-(L-M)(L+M)^{-1}\right)(L+M)^{-1} \xi=\frac{1}{2}(L+M)^{-1} \xi-\frac{1}{2}(L-M)(L+M)^{-2} \xi,
\end{gathered}
$$


but $(L+M)^{-1} \xi \in D(M)$ and, due to (10),

$$
(L-M)(L+M)^{-2} \xi \in D(M) .
$$

Finally $M \phi \in D(M)$ that is $\phi \in D\left(M^{2}\right)$. By exchange of the roles of $L$ and $M$, we get also $\phi \in D\left(L^{2}\right)$.

Conversely, let $\phi \in D\left(L^{2}\right) \cap D\left(M^{2}\right)$; then

$$
L \phi \in D(L)=D(L+M) \text { and } M \phi \in D(M)=D(L+M),
$$

so $(L+M) \phi \in D(L+M)$, that is $\phi \in D\left((L+M)^{2}\right)$.

3 . It is enough to combine (10) and statement 2 .

Lemma 2. Assume (6) (15). For any operator $S, \widetilde{S} \in\{L, M, L+M, L-M\}$, we have

1. $S(L-I)^{-1}, S(L+M)^{-1} \in \mathcal{L}(X)$,

2. $S(L-M)(L+M)^{-2}, S \widetilde{S}(L+M)^{-2} \in \mathcal{L}(X)$,

3. $(L+H) \Lambda^{-1},(M+H) \Lambda^{-1} \in \mathcal{L}(X)$.

The proof of the previous lemma is based on assumptions $(9) \sim(11)$ and the following remark: if $S$ is a closed linear operator on $X$ (or a sum of closed linear operators) and $W$ $\in \mathcal{L}(X)$ with $W(X) \subset D(S)$ then $S W \in \mathcal{L}(X)$.

Lemma 3. Assume (6) (13). Then

1. $(L \pm M)^{2}=L^{2} \pm L M \pm M L+M^{2}$ and for $\phi \in D\left((L+M)^{2}\right)$ we have

$$
(L \pm M)^{2} \phi=L^{2} \phi \pm L M \phi \pm M L \phi+M^{2} \phi,
$$

2. $L M+M L=\frac{1}{2}\left((L+M)^{2}-(L-M)^{2}\right)$,

3. $\left[(L-M) ;(L+M)^{-1}\right]=-2\left[M ;(L+M)^{-1}\right]=2\left[L ;(L+M)^{-1}\right]$,

4. $C_{L, M}=\frac{1}{2}(L+M)\left[(L-M) ;(L+M)^{-1}\right](L+M)^{-1}$,

5. $(L+H) \Lambda^{-1}=(L+M) \Lambda^{-1}+e^{L} e^{M}(L+H) \Lambda^{-1}-I$,

6. $(L+M)^{-1} M-(L+M)^{-1} C_{L, M}(L+M)=M(L+M)^{-1}$ and $(L+M)^{-1} L-(L+M)^{-1} C_{L, M}(L+M)=L(L+M)^{-1}$,

7. $M(L+M)^{-1} M+\frac{1}{2}(L-M)(L+M)^{-1} C_{L, M}(L+M)$

$$
\begin{aligned}
& =\frac{1}{2} M-\frac{1}{2}(L-M) M(L+M)^{-1} \text { and } \\
& L(L+M)^{-1} L+\frac{1}{2}(L-M)(L+M)^{-1} C_{L, M}(L+M) \\
& =\frac{1}{2} L+\frac{1}{2}(L-M) M(L+M)^{-1},
\end{aligned}
$$


8. $M+(L-M) M(L+M)^{-1}-(L M+M L)(L+M)^{-1}=0$ on $D(M)$, $L-(L-M) L(L+M)^{-1}-(L M+M L)(L+M)^{-1}=0$ on $D(L)$.

Proof. For Statements 1 and 2 see the proof of Lemma 2.2 in [2] and for statement 3 see Lemma 2.1 in [2].

Statement 4. For $\phi \in D\left((L+M)^{2}\right)$ we write

$$
\left\{\begin{array}{l}
(L+M)(L-M) \phi=L^{2} \phi-L M \phi+M L \phi-M^{2} \phi \\
(L-M)(L+M) \phi=L^{2} \phi+L M \phi-M L \phi-M^{2} \phi
\end{array}\right.
$$

then

$$
(L+M)(L-M) \phi-(L-M)(L+M) \phi=2(M L-L M) \phi,
$$

from which we deduce

$$
\begin{aligned}
& (L+M)\left[(L-M)(L+M)^{-1}-(L+M)^{-1}(L-M)\right](L+M)(L+M)^{-2} \\
& =2(M L-L M)(L+M)^{-2} .
\end{aligned}
$$

As we have mentioned it in the hypotheses, it follows that our commutator $C_{L, M}$ is well defined in $\mathcal{L}(X)$ and can be expressed as

$$
C_{L, M}=\frac{1}{2}(L+M)\left[(L-M) ;(L+M)^{-1}\right](L+M)^{-1} .
$$

Statement 5. It is enough to write

$$
\begin{aligned}
& (L+M) \Lambda^{-1}+e^{L} e^{M}(L+H) \Lambda^{-1}-I \\
& =\left[(L+M)+e^{L} e^{M}(L+H)-(M-H)-e^{L} e^{M}(L+H)\right] \Lambda^{-1}=(L+H) \Lambda^{-1} .
\end{aligned}
$$

Statement 6 . Due to statement 4 , we have

$$
(L+M)^{-1} C_{L, M}(L+M)=\frac{1}{2}\left[(L-M) ;(L+M)^{-1}\right],
$$

then, using statement 3 , we get

$$
\begin{gathered}
(L+M)^{-1} M-(L+M)^{-1} C_{L, M}(L+M)=(L+M)^{-1} M-\frac{1}{2}\left[(L-M) ;(L+M)^{-1}\right] \\
=(L+M)^{-1} M+\left[M ;(L+M)^{-1}\right]=M(L+M)^{-1} .
\end{gathered}
$$

The second equality is obtained in the same manner.

Statement 7. Due to statement 3 and (22), we have

$$
\begin{aligned}
& M(L+M)^{-1} M+\frac{1}{2}(L-M)(L+M)^{-1} C_{L, M}(L+M) \\
& =M(L+M)^{-1} M+\frac{1}{4}(L-M)\left[(L-M) ;(L+M)^{-1}\right] \\
& =M(L+M)^{-1} M-\frac{1}{2}(L-M)\left[M ;(L+M)^{-1}\right] \\
& =M(L+M)^{-1} M-\frac{1}{2}(L-M) M(L+M)^{-1}+\frac{1}{2}(L-M)(L+M)^{-1} M \\
& =M(L+M)^{-1} M-\frac{1}{2}(L-M) M(L+M)^{-1}+\frac{1}{2} M-M(L+M)^{-1} M \\
& =\frac{1}{2} M-\frac{1}{2}(L-M) M(L+M)^{-1} .
\end{aligned}
$$


Similarly, we obtain the second equality.

Statement 8. On the domain $D(L)=D(M)$, we have

$$
\begin{aligned}
& M+(L-M) M(L+M)^{-1}-(L M+M L)(L+M)^{-1} \\
= & M+(L-M) M(L+M)^{-1}+\frac{1}{2}\left((L-M)^{2}-(L+M)^{2}\right)(L+M)^{-1} \\
= & M+(L-M) M(L+M)^{-1}+\frac{1}{2}(L-M)^{2}(L+M)^{-1}-\frac{1}{2}(L+M) \\
= & (L-M) M(L+M)^{-1}+\frac{1}{2}(L-M)^{2}(L+M)^{-1}-\frac{1}{2}(L+M-2 M) \\
= & \frac{1}{2}(L-M)[2 M+(L-M)](L+M)^{-1}-\frac{1}{2}(L-M) \\
= & \frac{1}{2}(L-M)[M+L](L+M)^{-1}-\frac{1}{2}(L-M)=0 .
\end{aligned}
$$

The second equality is similarly obtained.

We need also the following classical results

Lemma 4. Assume (6) (8). Then, for

$$
f \in L^{p}(0,1, X), 1<p<+\infty \text { and } Q \in\{L, M\},
$$

we have

1. $x \mapsto Q \int_{0}^{x} e^{(x-s) Q} f(s) d s \in L^{p}(0,1, X)$,

2. $x \mapsto Q \int_{x}^{1} e^{(s-x) Q} f(s) d s \in L^{p}(0,1, X)$,

3. $x \mapsto Q \int_{0}^{1} e^{(x+s) Q} f(s) d s \in L^{p}(0,1, X)$,

4. $\int_{0}^{1} e^{s Q} f(s) d s, \int_{0}^{1} e^{(1-s) Q} f(s) d s \in(D(Q), X)_{\frac{1}{p}, p}$.

For statements 1, 2 and 3, see [8,9, p. 167-168] and also [10, (24), (25) and (26)]. Statement 4 is an easy consequence of statements 1 and 2, we proceed as in Remark 3 by using the fact that

$$
x \mapsto \int_{0}^{x} e^{(x-s) Q} f(s) d s, \quad x \mapsto \int_{x}^{1} e^{(s-x) Q} f(s) d s,
$$

belong to $W^{1, p}(0,1, X) \cap L^{p}(0,1, D(Q))$.

Lemma 5. Assume (6) (14) and let $\varphi \in(D(Q), X)_{\frac{1}{p}, p}$ with $Q \in\{L, M\}$. Then

1. $(L+M) \Lambda^{-1} \varphi \in(D(Q), X)_{\frac{1}{p}, p}$.

2. $(L+H) \Lambda^{-1} \varphi \in(D(Q), X)_{\frac{1}{p}, p}$. 
Proof. 1. Set $T=(L+M) \Lambda^{-1}$, then $T$ is a linear continuous operator from $X$ to $X$. But, due to (14), we obtain

$$
T(D(L)) \subset D(L) \text { and } T \in \mathcal{L}(D(L), D(L))
$$

(here $D(L)$ is a Banach space endowed with the graph norm). So, by the interpolation property we get

$$
T \in \mathcal{L}\left((X, D(L))_{\frac{1}{p}, p},(X, D(L))_{\frac{1}{p}, p}\right),
$$

see [11, p. 19].

2. Due to statement 5 of Lemma 3, we have

$$
(L+H) \Lambda^{-1}=(L+M) \Lambda^{-1}+e^{L} e^{M}(L+H) \Lambda^{-1}-I,
$$

then, from statement 1 , we deduce that $(L+H) \Lambda^{-1} \varphi \in(D(Q), X)_{\frac{1}{p}, p}$ for any $\varphi \in$ $(D(Q), X)_{\frac{1}{p}, p}$.

\section{Representation Formula}

Again, in this section, we drop $\omega$ in the notations. Assume that there exists a classical solution $u$ to $(3),(4)$. We want to find an integral equation satisfied by

$$
v(.):=(L+M)^{2} u(.)
$$

To this end, we begin par recalling that the solution in the commutative case writes in the following form

$$
\Phi+\Psi+d
$$

where, for $x \in[0,1]$

$$
\begin{aligned}
\Phi(x)= & (L+M)^{-1} \int_{0}^{x} e^{(x-s) M} f(s) d s+(L+M)^{-1} \int_{x}^{1} e^{(s-x) L} f(s) d s \\
\Psi(x)= & -(L+M)^{-1} e^{x M}(L+H) \Lambda^{-1} e^{L} \int_{0}^{1} e^{(1-s) M} f(s) d s \\
& +(L+M)^{-1} e^{x M}(L+H) \Lambda^{-1} \int_{0}^{1} e^{s L} f(s) d s \\
& -(L+M)^{-1} e^{(1-x) L} e^{M}(L+H) \Lambda^{-1} \int_{0}^{1} e^{s L} f(s) d s \\
& -(L+M)^{-1} e^{(1-x) L}\left[I-e^{M}(L+H) \Lambda^{-1} e^{L}\right] \int_{0}^{1} e^{(1-s) M} f(s) d s
\end{aligned}
$$

and

$$
\begin{aligned}
d(x)= & e^{x M} \Lambda^{-1} d_{0}-e^{(1-x) L} e^{M} \Lambda^{-1} d_{0}+e^{x M} e^{L}(L+H) \Lambda^{-1} u_{1} \\
& +e^{(1-x) L} u_{1}-e^{(1-x) L} e^{M} e^{L}(L+H) \Lambda^{-1} u_{1}
\end{aligned}
$$

See Cheggag et al. in [1, p. 63]. 
Now, in our non commutative case, since $u$ satisfies (3), for almost all $x \in(0,1)$, we have

$$
\begin{aligned}
& \Phi(x)=(L+M)^{-1} \int_{0}^{x} e^{(x-s) M}\left[u^{\prime \prime}(s)+(L-M) u^{\prime}(s)-\frac{1}{2}(L M+M L) u(s)\right] d s \\
& +(L+M)^{-1} \int_{x}^{1} e^{(s-x) L}\left[u^{\prime \prime}(s)+(L-M) u^{\prime}(s)-\frac{1}{2}(L M+M L) u(s)\right] d s .
\end{aligned}
$$

Set $\Phi=\sum_{i=1}^{6} \Phi_{i}$ where

$$
\begin{aligned}
& \Phi_{1}(x)=(L+M)^{-1} \int_{0}^{x} e^{(x-s) M} u^{\prime \prime}(s) d s \\
& \Phi_{2}(x)=(L+M)^{-1} \int_{x}^{1} e^{(s-x) L} u^{\prime \prime}(s) d s \\
& \Phi_{3}(x)=(L+M)^{-1} \int_{0}^{x} e^{(x-s) M}(L-M) u^{\prime}(s) d s \\
& \Phi_{4}(x)=(L+M)^{-1} \int_{x}^{1} e^{(s-x) L}(L-M) u^{\prime}(s) d s \\
& \Phi_{5}(x)=-\frac{1}{2}(L+M)^{-1} \int_{0}^{x} e^{(x-s) M}(L M+M L) u(s) d s \\
& \Phi_{6}(x)=-\frac{1}{2}(L+M)^{-1} \int_{x}^{1} e^{(s-x) L}(L M+M L) u(s) d s
\end{aligned}
$$

then the main idea is to carry out integration by parts in view to deduce the integral equation satisfied by $\Phi$. Since $u$ satisfies (5), all the above calculations are justified for almost all $x \in(0,1)$. Then

$$
\begin{aligned}
& \Phi_{1}(x)=(L+M)^{-1} \int_{0}^{x} e^{(x-s) M} u^{\prime \prime}(s) d s=(L+M)^{-1} u^{\prime}(x)-(L+M)^{-1} e^{x M} u^{\prime}(0) \\
& +(L+M)^{-1} M u(x)-(L+M)^{-1} M e^{x M} u(0)+(L+M)^{-1} \int_{0}^{x} M^{2} e^{(x-s) M} u(s) d s,
\end{aligned}
$$

here we have used the fact that, for any $g \in W^{1, p}(0,1 ; X)$

$$
g(x)-g(0)=\int_{0}^{x} g^{\prime}(s) d s .
$$

Similarly, we have

$$
\begin{gathered}
\Phi_{2}(x)=(L+M)^{-1} \int_{x}^{1} e^{(s-x) L} u^{\prime \prime}(s) d s=(L+M)^{-1} e^{(1-x) L} u^{\prime}(1)-(L+M)^{-1} u^{\prime}(x) \\
-(L+M)^{-1} L e^{(1-x) L} u_{1}+(L+M)^{-1} L u(x)+(L+M)^{-1} \int_{x}^{1} L^{2} e^{(s-x) L} u(s) d s .
\end{gathered}
$$

Due to $(19), u(0), u_{1} \in D(L)=D(M)$; so

$$
\begin{aligned}
(L+M)^{-1} M e^{x M} u(0) & =(L+M)^{-1} e^{x M} M u(0), \\
(L+M)^{-1} L e^{(1-x) L} u_{1} & =(L+M)^{-1} e^{(1-x) L} L u_{1} .
\end{aligned}
$$


Since $v()=.(L+M)^{2} u($.$) and u^{\prime}(0)=d_{0}+H u(0)$, we get

$$
\begin{gathered}
(L+M)^{2}\left(\Phi_{1}(x)+\Phi_{2}(x)\right)=v(x)-(L+M) e^{x M}(H+M) u(0) \\
+(L+M) e^{(1-x) L}\left(u^{\prime}(1)-L u_{1}\right)-(L+M) e^{x M} d_{0} \\
+(L+M) \int_{0}^{x} M^{2} e^{(x-s) M}(L+M)^{-2} v(s) d s+(L+M) \int_{x}^{1} L^{2} e^{(s-x) L}(L+M)^{-2} v(s) d s .
\end{gathered}
$$

In the same manner, for $\Phi_{3}(x)+\Phi_{4}(x)$, the integration by parts gives

$$
\begin{aligned}
& \Phi_{3}(x)+\Phi_{4}(x)=-(L+M)^{-1} e^{x M}(L-M) u(0)+(L+M)^{-1} e^{(1-x) L}(L-M) u(1) \\
& +(L+M)^{-1} \int_{0}^{x} M e^{(x-s) M}(L-M) u(s) d s-(L+M)^{-1} \int_{x}^{1} L e^{(s-x) L}(L-M) u(s) d s
\end{aligned}
$$

but

$$
\left\{\begin{array}{l}
M(L-M) u(x)=M(L-M)(L+M)^{-2}(L+M)^{2} u(x), \\
L(L-M) u(x)=L(L-M)(L+M)^{-2}(L+M)^{2} u(x),
\end{array}\right.
$$

So

$$
\begin{gathered}
(L+M)\left(\Phi_{3}(x)+\Phi_{4}(x)\right)=-e^{x M}(L-M) u(0)+e^{(1-x) L}(L-M) u_{1} \\
+\int_{0}^{x} e^{(x-s) M} M(L-M)(L+M)^{-2} v(s) d s-\int_{x}^{1} e^{(s-x) L} L(L-M)(L+M)^{-2} v(s) d s .
\end{gathered}
$$

Therefore, applying again $L+M$, we get

$$
\begin{gathered}
(L+M)^{2}\left(\Phi_{3}(x)+\Phi_{4}(x)\right)-(L+M) e^{x M}(L-M) u(0) \\
+(L+M) e^{(1-x) L}(L-M) u_{1}+(L+M) \int_{0}^{x} e^{(x-s) M} M L(L+M)^{-2} v(s) d s \\
+(L+M) \int_{x}^{1} e^{(s-x) L} L M(L+M)^{-2} v(s) d s \\
-(L+M) \int_{0}^{x} e^{(x-s) M} M^{2}(L+M)^{-2} v(s) d s-(L+M) \int_{x}^{1} e^{(s-x) L} L^{2}(L+M)^{-2} v(s) d s .
\end{gathered}
$$

Finally

$$
\begin{gathered}
(L+M)\left(\Phi_{5}(x)+\Phi_{6}(x)\right)=-\frac{1}{2} \int_{0}^{x} e^{(x-s) M} L M(L+M)^{-2} v(s) d s \\
-\frac{1}{2} \int_{0}^{x} e^{(x-s) M} M L(L+M)^{-2} v(s) d s-\frac{1}{2} \int_{x}^{1} e^{(s-x) L} L M(L+M)^{-2} v(s) d s \\
-\frac{1}{2} \int_{x}^{1} e^{(s-x) L} M L(L+M)^{-2} v(s) d s
\end{gathered}
$$

it follows that

$$
\begin{gathered}
(L+M)^{2}\left(\Phi_{5}(x)+\Phi_{6}(x)\right)=-\frac{1}{2}(L+M) \int_{0}^{x} e^{(x-s) M} L M(L+M)^{-2} v(s) d s \\
-\frac{1}{2}(L+M) \int_{0}^{x} e^{(x-s) M} M L(L+M)^{-2} v(s) d s \\
-\frac{1}{2}(L+M) \int_{x}^{1} e^{(s-x) L} L M(L+M)^{-2} v(s) d s \\
-\frac{1}{2}(L+M) \int_{x}^{1} e^{(s-x) L} M L(L+M)^{-2} v(s) d s .
\end{gathered}
$$

Then, using (27), (28) and (29), we have

$$
\begin{gathered}
(L+M)^{2} \Phi(x)=v(x)-(L+M) e^{x M}\left((H+L) u(0)+d_{0}\right) \\
+(L+M) e^{(1-x) L}\left(u^{\prime}(1)-M u_{1}\right)+\frac{1}{2}(L+M) \int_{0}^{x} e^{(x-s) M} M L(L+M)^{-2} v(s) d s \\
-\frac{1}{2}(L+M) \int_{0}^{x} e^{(x-s) M} L M(L+M)^{-2} v(s) d s \\
+\frac{1}{2}(L+M) \int_{x}^{1} e^{(s-x) L} L M(L+M)^{-2} v(s) d s-\frac{1}{2}(L+M) \int_{x}^{1} e^{(s-x) L} M L(L+M)^{-2} v(s) d s .
\end{gathered}
$$


We deduce the following integral equation

$$
\begin{aligned}
(L+M)^{2} \Phi(x) & =v(x)-(L+M) e^{x M}\left[(H+L) u(0)+d_{0}\right] \\
& +(L+M) e^{(1-x) L}\left(u^{\prime}(1)-M u_{1}\right) \\
& +\frac{1}{2}(L+M) \int_{0}^{x} e^{(x-s) M} C_{L, M} v(s) d s \\
& -\frac{1}{2}(L+M) \int_{x}^{1} e^{(s-x) L} C_{L, M} v(s) d s
\end{aligned}
$$

where

$$
C_{L, M}:=[M ; L](L+M)^{-2}=(M L-L M)(L+M)^{-2} .
$$

Concerning $\Psi$, by the same method, we obtain the following integral equation

$$
\begin{aligned}
& (L+M)^{2} \Psi(x) \\
= & (L+M) e^{x M}(L+H) u(0)-(L+M) e^{(1-x) L} u^{\prime}(1) \\
& -(L+M) e^{x M}(L+H) \Lambda^{-1} e^{L}(L+M) u_{1} \\
& -(L+M) e^{x M}(L+H) \Lambda^{-1}\left(I-e^{L} e^{M}\right) d_{0} \\
& +(L+M) e^{(1-x) M}(L+H) \Lambda^{-1} e^{L}(L+M) u_{1} \\
& +(L+M) e^{(1-x) L} e^{M}(L+H) \Lambda^{-1}\left(I-e^{L} e^{M}\right) d_{0} \\
& -(L+M) e^{(1-x) L} L u_{1}+(L+M) e^{(1-x) L} e^{M} d_{0} \\
& -\frac{1}{2}(L+M) e^{x M}(L+H) \Lambda^{-1} e^{L} \int_{0}^{1} e^{(1-s) M} C_{L, M} v(s) d s \\
& -(L+M) e^{x M}(L+H) \Lambda^{-1} \int_{0}^{1} e^{s L} C_{L, M} v(s) d s \\
& +\frac{1}{2}(L+M) e^{(1-x) L} e^{M}(L+H) \Lambda^{-1} \int_{0}^{1} e^{s L} C_{L, M} v(s) d s \\
& -\frac{1}{2}(L+M) e^{(1-x) L}\left[I-e^{M}(L+H) \Lambda^{-1} e^{L}\right] \int_{0}^{1} e^{(1-s) M} C_{L, M} v(s) d s .
\end{aligned}
$$

This last equation, together with (30), leads us to set

$$
v+R(v)=F(f)+\Gamma,
$$

where for all $x \in] 0,1[$

$$
\begin{aligned}
& R(v)(x) \\
= & \frac{1}{2}(L+M) \int_{0}^{x} e^{(x-s) M} C_{L, M} v(s) d s-\frac{1}{2}(L+M) \int_{x}^{1} e^{(s-x) L} C_{L, M} v(s) d s \\
& -\frac{1}{2}(L+M) e^{x M}(L+H) \Lambda^{-1} \int_{0}^{1} e^{s L} C_{L, M} v(s) d s \\
& -\frac{1}{2}(L+M) e^{x M}(L+H) \Lambda^{-1} e^{L} \int_{0}^{1} e^{(1-s) M} C_{L, M} v(s) d s \\
& -\frac{1}{2}(L+M) e^{(1-x) L}\left[I-e^{M}(L+H) \Lambda^{-1} e^{L}\right] \int_{0}^{1} e^{(1-s) M} C_{L, M} v(s) d s \\
& +\frac{1}{2}(L+M) e^{(1-x) L} e^{M}(L+H) \Lambda^{-1} \int_{0}^{1} e^{s L} C_{L, M} v(s) d s,
\end{aligned}
$$




$$
\begin{aligned}
& F(f)(x) \\
= & (L+M) \int_{0}^{x} e^{(x-s) M} f(s) d s+(L+M) \int_{x}^{1} e^{(s-x) L} f(s) d s \\
& -(L+M) e^{x M}(L+H) \Lambda^{-1} e^{L} \int_{0}^{1} e^{(1-s) M} f(s) d s \\
& +(L+M) e^{x M}(L+H) \Lambda^{-1} \int_{0}^{1} e^{s L} f(s) d s \\
& -(L+M) e^{(1-x) L} e^{M}(L+H) \Lambda^{-1} \int_{0}^{1} e^{s L} f(s) d s \\
& -(L+M) e^{(1-x) L}\left[I-e^{M}(L+H) \Lambda^{-1} e^{L}\right] \int_{0}^{1} e^{(1-s) M} f(s) d s,
\end{aligned}
$$

and

$$
\begin{aligned}
\Gamma(x)= & (L+M) e^{x M}(L+H) \Lambda^{-1}\left(I-e^{L} e^{M}\right) d_{0} \\
& +(L+M) e^{x M} d_{0}-(L+M) e^{(1-x) L} e^{M} d_{0} \\
& -(L+M) e^{(1-x) L} e^{M}(L+H) \Lambda^{-1}\left(I-e^{L} e^{M}\right) d_{0} \\
& +(L+M) e^{x M}(L+H) \Lambda^{-1} e^{L}(L+M) u_{1} \\
& -(L+M) e^{(1-x) L} e^{M}(L+H) \Lambda^{-1} e^{L}(L+M) u_{1} \\
& +(L+M) e^{(1-x) L}(L+M) u_{1} .
\end{aligned}
$$

Finally, if $u$ is a classical solution of (3), (4), then $v():.=(L+M)^{2} u($.$) satisfies the integral$ equation

$$
v+R(v)=F(f)+\Gamma,
$$

with $R, F(f)$ and $\Gamma$ are defined by (32), (33) and (34) respectively.

Remark 5. Since

$$
(L+H) \Lambda^{-1}=(L+M) \Lambda^{-1}+e^{L} e^{M}(L+H) \Lambda^{-1}-I,
$$

we can write $\Gamma(x)=\widetilde{R}(x)+S(x)$, where

$$
\begin{aligned}
\widetilde{R}(x)= & (L+M) e^{x M} e^{L} e^{M}(L+H) \Lambda^{-1} d_{0} \\
& -(L+M) e^{x M}(L+M) \Lambda^{-1} e^{L} e^{M} d_{0} \\
& -(L+M) e^{x M} e^{L} e^{M}(L+H) \Lambda^{-1} e^{L} e^{M} d_{0} \\
& +(L+M) e^{x M} e^{L} e^{M} d_{0} \\
& -(L+M) e^{(1-x) L} e^{M}\left[I+(L+H) \Lambda^{-1}\left(I-e^{L} e^{M}\right)\right] d_{0} \\
& +(L+M) e^{x M} e^{L} e^{M}(L+H) \Lambda^{-1} e^{L}(L+M) u_{1} \\
& -(L+M) e^{x M} e^{L}(L+M) u_{1} \\
& -(L+M) e^{(1-x) L} e^{M}(L+H) \Lambda^{-1} e^{L}(L+M) u_{1} \\
& +(L+M) e^{x M}(L+M) \Lambda^{-1} e^{L}(L+M) u_{1},
\end{aligned}
$$

and

$$
S(x)=(L+M) e^{x M}(L+M) \Lambda^{-1} d_{0}+(L+M) e^{(1-x) L}(L+M) u_{1} .
$$


$\widetilde{R}$ will be regular since it corresponds to the terms of $\Gamma$ containing $e^{L}$ or $e^{M}$. Then, to obtain a uniqueness and existence result for our problem, it will be sufficient to study the regularity of $R, F(f)$ and $S$.

\section{Study of $F_{\omega}(f), S_{\omega}, \widetilde{R_{\omega}}$ and $R_{\omega}$}

Suppose that $u_{1} \in D(L+M)=D(L)$.

Let us recall the following result (see [12, p. 96]):

Proposition 1. Let $Q$ the infinitesimal generator of an analytic semigroup in $X$, $\left(e^{x Q}\right)_{x \geq 0}, \varphi \in X, 1<p<\infty$ and $m \in \mathbb{N} \backslash\{0\}$. Then

1. $e^{Q} \varphi \in L^{p}(0,1 ; X)$,

2. $Q^{m} e^{\cdot Q} \varphi \in L^{p}(0,1 ; X) \Leftrightarrow e^{\cdot Q} \varphi \in W^{m, p}(0,1 ; X) \Leftrightarrow \varphi \in\left(X, D\left(Q^{m}\right)\right)_{1-\frac{1}{m p}, p}$.

Set, for a given function $g$ from $(0,1)$ to $X$ and for $x \in(0,1)$

$$
G(g)(x)=\int_{0}^{x} e^{(x-s) M} g(s) d s, \quad K(g)(x)=\int_{x}^{1} e^{(s-x) L} g(s) d s .
$$

Applying [4], a remark in [13, p. 25] and using the closed graph theorem, we obtain Proposition 2. Assume (6) (9). Let $g \in L^{p}(0,1 ; X), 1<p<\infty$. Then

$$
K(g), G(g) \in W^{1, p}(0,1 ; X) \cap L^{p}(0,1 ; D(M)),
$$

and this implies that

$$
G(g)(1), K(g)(0) \in(D(M), X)_{\frac{1}{p}, p}=(D(L), X)_{\frac{1}{p}, p} .
$$

Moreover, for a.e. $x \in(0,1)$

$$
G^{\prime}(g)(x)=M G(g)(x)+g(x) \text { and } K^{\prime}(g)(x)=-L K(g)(x)-g(x),
$$

and there exists $C>0$ such that

$$
\left\{\begin{array}{l}
\|G(g)\|_{L^{p}(0,1 ; X)} \leqslant C\|g\|_{L^{p}(0,1 ; X)},\|K(g)\|_{L^{p}(0,1 ; X)} \leqslant C\|g\|_{L^{p}(0,1 ; X)}, \\
\|x \mapsto M G(g)(x)\|_{L^{p}(0,1 ; X)} \leqslant C\|g\|_{L^{p}(0,1 ; X)}, \\
\|x \mapsto L K(g)(x)\|_{L^{p}(0,1 ; X)} \leqslant C\|g\|_{L^{p}(0,1 ; X)} .
\end{array}\right.
$$

Proposition 3. Assume (6) (9) and (13) (14). Let $g \in L^{p}(0,1 ; X)$ with $1<p<\infty$. Then the following functions

$$
\begin{aligned}
& \Psi_{1}(g):=e^{\cdot M}(L+H) \Lambda^{-1} K(g)(0), \\
& \Psi_{2}(g):=e^{\cdot M}(L+H) \Lambda^{-1} e^{L} G(g)(1) \\
& \Psi_{3}(g):=e^{(1-.) L} e^{M}(L+H) \Lambda^{-1} e^{L} G(g)(1), \\
& \Psi_{4}(g):=e^{(1-\cdot) L} e^{M}(L+H) \Lambda^{-1} K(g)(0), \\
& \Psi_{5}(g):=e^{(1-\cdot) L} G(g)(1),
\end{aligned}
$$


belong to $L^{p}(0,1 ; D(L))$ and there exists $C>0$, such that

$$
\left\{\begin{array}{l}
\left\|\Psi_{i}(g)\right\|_{L^{p}(0,1 ; X)} \leq C\|g\|_{L^{p}(0,1 ; X)}, i=1, . ., 5 \\
\left\|x \mapsto M \Psi_{i}(g)(x)\right\|_{L^{p}(0,1 ; X)} \leq C\|g\|_{L^{p}(0,1 ; X)}, i=1,2 \\
\left\|x \mapsto L \Psi_{i}(g)(x)\right\|_{L^{p}(0,1 ; X)} \leq C\|g\|_{L^{p}(0,1 ; X)}, i=3,4,5
\end{array}\right.
$$

Proof. We study only $\Psi_{1}(g)$ (the other functions are similarly treated). In the following, $C$ denotes various constants which can depend on $p$ but do not depend on $g$ ). Clearly $\Psi_{1}(g) \in L^{p}(0,1 ; X)$ and $\left\|\Psi_{1}(g)\right\|_{L^{p}(0,1 ; X)} \leq C\|g\|_{L^{p}(0,1 ; X)}$. Let us show that $\Psi_{1}(g) \in$ $L^{p}(0,1 ; D(M))$. We have

$$
\begin{aligned}
\Psi_{1}(g)(x) & =e^{x M}(L+H) \Lambda^{-1} \int_{0}^{1} e^{s L} g(s) d s \\
& =\int_{0}^{x} e^{(x-s) M} e^{s M}(L+H) \Lambda^{-1} e^{s L} g(s) d s+e^{x M}(L+H) \Lambda^{-1} \int_{x}^{1} e^{s L} g(s) d s
\end{aligned}
$$

that is $\Psi_{1}(g)(x)=G(\widetilde{g})(x)+\widetilde{\Psi}_{1}(g)(x)$, where

$$
\widetilde{g}(s)=e^{s M}(L+H) \Lambda^{-1} e^{s L} g(s) \text { and } \widetilde{\Psi}_{1}(g)=e^{x M}(L+H) \Lambda^{-1} \int_{x}^{1} e^{s L} g(s) d s .
$$

But $\widetilde{g} \in L^{p}(0,1 ; X)$, so due to Proposition $2, G(\widetilde{g}) \in L^{p}(0,1 ; D(M))$ and

$$
\|x \mapsto M G(\widetilde{g})(x)\|_{L^{p}(0,1 ; X)} \leqslant C\|\widetilde{g}\|_{L^{p}(0,1 ; X)} \leqslant C\|g\|_{L^{p}(0,1 ; X)} .
$$

Now, from Lemma 3, statement 5, we have

$$
(L+H) \Lambda^{-1}=(L+M) \Lambda^{-1}+e^{L} e^{M}(L+H) \Lambda^{-1}-I,
$$

and then

$$
\begin{aligned}
\widetilde{\Psi}_{1}(g)(x) & =e^{x M}(L+M) \Lambda^{-1} \int_{x}^{1} e^{s L} g(s) d s \\
& +e^{x M} e^{L} e^{M}(L+H) \Lambda^{-1} \int_{x}^{1} e^{s L} g(s) d s-e^{x M} \int_{x}^{1} e^{s L} g(s) d s \\
& =I_{1}(x)+I_{2}(x)+I_{3}(x) .
\end{aligned}
$$

Since $e^{L}(X) \subset D(M)$ we have $M e^{L} e^{M}(L+H) \Lambda^{-1} \in \mathcal{L}(X)$ and we can write

$$
\begin{aligned}
\int_{0}^{1}\left\|M I_{2}(x)\right\|^{p} d x & =\int_{0}^{1}\left\|e^{x M} M e^{L} e^{M}(L+H) \Lambda^{-1} e^{x L} \int_{x}^{1} e^{(s-x) L} g(s) d s\right\|^{p} d x \\
& \leqslant C \int_{0}^{1}\left\|\int_{x}^{1} e^{(s-x) L} g(s) d s\right\|^{p} d x \leqslant C\|K(g)\|_{L^{p}(0,1 ; X)}^{p} \leqslant C\|g\|_{L^{p}(0,1 ; X)}^{p},
\end{aligned}
$$

similarly

$$
\begin{gathered}
\int_{0}^{1}\left\|M I_{3}(x)\right\|^{p} d x=\int_{0}^{1}\left\|e^{x M} M(L-I)^{-1}(L-I) \int_{x}^{1} e^{s L} g(s) d s\right\|^{p} d x \\
\leqslant C \int_{0}^{1}\left\|(L-I) \int_{x}^{1} e^{s L} g(s) d s\right\|^{p} d x \leqslant C\left(\|L K(g)\|_{L^{p}(0,1 ; X)}+\|K(g)\|\right)^{p} \leqslant C\|g\|_{L^{p}(0,1 ; X)}^{p} .
\end{gathered}
$$


For $I_{1}$, we use (14) which shows that $M(L+M) \Lambda^{-1}(L-I)^{-1} \in \mathcal{L}(X)$ and then

$$
\begin{gathered}
\int_{0}^{1}\left\|M I_{1}(x)\right\|^{p} d x=\int_{0}^{1}\left\|e^{x M} M(L+M) \Lambda^{-1}(L-I)^{-1}(L-I) \int_{x}^{1} e^{s L} g(s) d s\right\|^{p} d x \\
\leqslant C \int_{0}^{1}\left\|(L-I) \int_{x}^{1} e^{s L} g(s) d s\right\|^{p} d x \leqslant C\|g\|_{L^{p}(0,1 ; X)}^{p} .
\end{gathered}
$$

Finally, the previous estimates concerning $I_{1}, I_{2}$ and $I_{3}$ together with (37) and (38) prove that $\Psi_{1}(g) \in L^{p}(0,1 ; D(M))$ and

$$
\left\|x \mapsto M \Psi_{1}(g)(x)\right\|_{L^{p}(0,1 ; X)} \leq C\|g\|_{L^{p}(0,1 ; X)} .
$$

Proposition 4. Assume (6) (14). Let $f \in L^{p}(0,1 ; X), 1<p<\infty$. Then

$$
F(f) \in L^{p}(0,1 ; X) .
$$

Proof. Using Propositions 2 and 3, we can write

$$
\begin{aligned}
F(f)(.) & =(L+M)(M-I)^{-1}(M-I) G(f)+(L+M)(L-I)^{-1}(L-I)[K(f)(.)] \\
& -(L+M)(M-I)^{-1}(M-I) \Psi_{2}(f)+(L+M)(M-I)^{-1}(M-I)\left[\Psi_{1}(f)(.)\right] \\
& -(L+M)(L-I)^{-1}(L-I) \Psi_{4}(f)+(L+M)(L-I)^{-1}(L-I)\left[\Psi_{3}(f)(.)\right] \\
& -(L+M)(L-I)^{-1}(L-I)\left[\Psi_{5}(f)(.)\right] .
\end{aligned}
$$

$\operatorname{But}(L+M)(M-I)^{-1},(L+M)(L-I)^{-1} \in \mathcal{L}(X)$. So $F(f) \in L^{p}(0,1 ; X)$.

Proposition 5. Assume (6) (14). Let $1<p<\infty$. Then $\widetilde{R} \in L^{p}(0,1 ; X)$.

Proof. Set

$$
U:=(L+M)(L-I)^{-1} \in \mathcal{L}(X) \text { and } V:=(L+M)(M-I)^{-1} \in \mathcal{L}(X) .
$$

It is well known that, for any $n \in \mathbb{N}^{*}$ and any $\xi \in X$, we have

$$
e^{L} \xi \in D\left(L^{n}\right) \text { and } e^{M} \xi \in D\left(M^{n}\right),
$$

thus $(M-I) e^{L},(L-I) e^{M} \in \mathcal{L}(X)$ and also, using hypothesis (14),

$$
(M-I)(L+M) \Lambda^{-1} e^{L}=M(L+M) \Lambda^{-1}(L-I)^{-1}(L-I) e^{L}-(L+M) \Lambda^{-1} e^{L} \in \mathcal{L}(X) .
$$

For $x \in(0,1)$, we can write

$$
\begin{aligned}
\widetilde{R}(x) & =V e^{x M}(M-I) e^{L} e^{M}(L+H) \Lambda^{-1} d_{0}-V e^{x M}(M-I)(L+M) \Lambda^{-1} e^{L} e^{M} d_{0} \\
& -V e^{x M}(M-I) e^{L} e^{M}(L+H) \Lambda^{-1} e^{L} e^{M} d_{0}+V e^{x M}(M-I) e^{L} e^{M} d_{0} \\
& -U e^{(1-x) L}(L-I) e^{M}\left[I+(L+H) \Lambda^{-1}\left(I-e^{L} e^{M}\right)\right] d_{0} \\
& +V e^{x M}(M-I) e^{L} e^{M}(L+H) \Lambda^{-1} e^{L}(L+M) u_{1}-V e^{x M}(M-I) e^{L}(L+M) u_{1} \\
& -U e^{(1-x) L}(L-I) e^{M}(L+H) \Lambda^{-1} e^{L}(L+M) u_{1} \\
& +V e^{x M}(M-I)(L+M) \Lambda^{-1} e^{L}(L+M) u_{1} .
\end{aligned}
$$

Then, from Proposition 1 , statement 1 , we obtain $\widetilde{R} \in L^{p}(0,1 ; X)$. 
Proposition 6. Assume (6) (13). Let $1<p<\infty$. Then $S \in L^{p}(0,1 ; X)$ if and only if $u_{1}, \Lambda^{-1} d_{0} \in\left(X, D\left((L+M)^{2}\right)\right)_{1-\frac{1}{2 p}, p}$.

Proof. For $x \in(0,1)$, we have

$$
\begin{aligned}
S(x) & =(L+M) e^{x M}(L+M) \Lambda^{-1} d_{0}+(L+M) e^{(1-x) L}(L+M) u_{1} \\
& =(L+M)(M-I)^{-1}(M-I) e^{x M}(L+M) \Lambda^{-1} d_{0} \\
& +(L+M)(L-I)^{-1}(L-I) e^{(1-x) L}(L+M) u_{1}=S_{1}(x)+S_{2}(x) .
\end{aligned}
$$

Since $(L+M)(M-I)^{-1}$ is invertible in $\mathcal{L}(X)$, then $S_{1} \in L^{p}(0,1 ; X)$ if and only if

$$
x \rightarrow(M-I) e^{x M}(L+M) \Lambda^{-1} d_{0} \in L^{p}(0,1 ; X),
$$

and this, from Proposition 1, is equivalent to

$$
(L+M) \Lambda^{-1} d_{0} \in(X, D(M))_{1-\frac{1}{p}, p}=(X, D(L+M))_{1-\frac{1}{p}, p} .
$$

Similarly, $S_{2} \in L^{p}(0,1 ; X)$ if and only if

$$
(L+M) u_{1} \in(X, D(L))_{1-\frac{1}{p}, p}=(X, D(L+M))_{1-\frac{1}{p}, p} .
$$

We have $S_{1} \in L^{p}\left(\frac{1}{2}, 1 ; X\right)$ and $S_{2} \in L^{p}\left(0, \frac{1}{2} ; X\right)$; then

$$
\begin{aligned}
S \in L^{p}(0,1 ; X) & \Longleftrightarrow S \in L^{p}\left(0, \frac{1}{2} ; X\right) \text { and } S \in L^{p}\left(\frac{1}{2}, 1 ; X\right) \\
& \Longleftrightarrow S_{1} \in L^{p}\left(0, \frac{1}{2} ; X\right) \text { and } S_{2} \in L^{p}\left(\frac{1}{2}, 1 ; X\right) \\
& \Longleftrightarrow\left\{\begin{array}{l}
(L+M) \Lambda^{-1} d_{0} \in(X, D(L+M))_{1-\frac{1}{p}, p} \text { and } \\
(L+M) u_{1} \in(X, D(L+M))_{1-\frac{1}{p}, p},
\end{array}\right.
\end{aligned}
$$

and, by using the reiteration property, we then get

$$
S \in L^{p}(0,1 ; X) \Longleftrightarrow u_{1}, \Lambda^{-1} d_{0} \in\left(X, D\left((L+M)^{2}\right)\right)_{1-\frac{1}{2 p}, p} .
$$

Now, we need the dependence of $\omega$. Operators $R, \widetilde{R}, F(f)$ and $S$ become $R_{\omega}, \widetilde{R}_{\omega}, F_{\omega}(f)$ and $S_{\omega}$ where $M$ and $L$ are replaced by $M_{\omega}$ and $L_{\omega}$.

We must estimate $\left\|R_{\omega}\right\|$ in order to invert $I+R_{\omega}$ for $\omega$ large enough and obtain the following representation formula for the classical solution $u$ to $(3)$, (4):

$$
u(.)=\left(L_{\omega}+M_{\omega}\right)^{-2}\left[\left(I+R_{\omega}\right)^{-1}\left(F_{\omega}(f)+\widetilde{R}_{\omega}+S_{\omega}\right)(.)\right] .
$$

Proposition 7. Assume (6) (16). Let $1<p<+\infty$. Then $R_{\omega} \in \mathcal{L}\left(L^{p}(0,1 ; X)\right)$ and there exits $\omega^{*} \geqslant \omega_{0}$ such that, for each $\omega \geqslant \omega^{*}, I+R_{\omega}$ is invertible in $\mathcal{L}\left(L^{p}(0,1 ; X)\right)$.

Proof. Let $v \in L^{p}(0,1 ; X)$. Since $C_{L_{\omega}, M_{\omega}} \in \mathcal{L}(X)$ then $C_{L_{\omega}, M_{\omega}} v \in L^{p}(0,1 ; X)$. Using the notation of Propositions 2 and 3 , we can write

$$
\begin{aligned}
2 R_{\omega}(v)(.) & =\left(L_{\omega}+M_{\omega}\right)\left(M_{\omega}-I\right)^{-1}\left(M_{\omega}-I\right) G\left(C_{L_{\omega}, M_{\omega}} v(.)\right)(.) \\
& -\left(L_{\omega}+M_{\omega}\right)\left(L_{\omega}-I\right)^{-1}\left(L_{\omega}-I\right) K\left(C_{L_{\omega}, M_{\omega}} v(.)\right)(.) \\
& -\left(L_{\omega}+M_{\omega}\right)\left(M_{\omega}-I\right)^{-1}\left(M_{\omega}-I\right) \Psi_{1}\left(C_{L_{\omega}, M_{\omega}} v(.)\right)(.) \\
& -\left(L_{\omega}+M_{\omega}\right)\left(M_{\omega}-I\right)^{-1}\left(M_{\omega}-I\right) \Psi_{2}\left(C_{L_{\omega}, M_{\omega}} v(.)\right)(.) \\
& +\left(L_{\omega}+M_{\omega}\right)\left(L_{\omega}-I\right)^{-1}\left(L_{\omega}-I\right) \Psi_{3}\left(C_{L_{\omega}, M_{\omega}} v(.)\right)(.) \\
& +\left(L_{\omega}+M_{\omega}\right)\left(L_{\omega}-I\right)^{-1}\left(L_{\omega}-I\right) \Psi_{4}\left(C_{L_{\omega}, M_{\omega}} v(.)\right)(.) \\
& -\left(L_{\omega}+M_{\omega}\right)\left(L_{\omega}-I\right)^{-1}\left(L_{\omega}-I\right) \Psi_{5}\left(C_{L_{\omega}, M_{\omega}} v(.)\right)(.) .
\end{aligned}
$$


Recall that $\left(L_{\omega}+M_{\omega}\right)\left(M_{\omega}-I\right)^{-1},-\left(L_{\omega}+M_{\omega}\right)\left(L_{\omega}-I\right)^{-1} \in \mathcal{L}(X)$. Then, due to Propositions 2 and 3 , we have $R_{\omega}(v) \in L^{p}(0,1 ; X)$ and there exists a constant $b>0$ such that, for every $\omega \geqslant \omega_{0}$

$$
\left\|R_{\omega}(v)\right\|_{L^{p}(0,1 ; X)} \leqslant b\left\|C_{L_{\omega}, M_{\omega}}\right\|_{\mathcal{L}(X)}\|v\|_{L^{p}(0,1 ; X)},
$$

and by hypothesis (16), we have

$$
\left\|R_{\omega}(v)\right\|_{L^{p}(0,1 ; X)} \leqslant C \chi(\omega)\|v\|_{L^{p}(0,1 ; X)},
$$

with $\lim _{\omega \rightarrow+\infty} \chi(\omega)=0$. Therefore, there exists $\omega^{*} \geqslant \omega_{0}$ such that for every $\omega \geqslant \omega^{*}$

$$
\left\|R_{\omega}\right\|_{\mathcal{L}\left(L^{p}(0,1 ; X)\right)}<1 .
$$

So $I+R_{\omega}$ is invertible in $\mathcal{L}\left(L^{p}(0,1 ; X)\right)$ for any $\omega \geqslant \omega^{*}$.

\section{Proof of Theorem 1}

We consider the fixed number $\omega^{*}$ built above and let $\omega \geqslant \omega^{*}$.

\subsection{Statement 1 Implies Statement 2}

Assume statement 1 , that is $u$, defined by (39), is the classical solution of (3), (4). Then

$$
x \mapsto\left(L_{\omega}+M_{\omega}\right)^{2} u(x)=\left(I+R_{\omega}\right)^{-1}\left(F_{\omega}(f)(x)+\widetilde{R}_{\omega}(x)+S_{\omega}(x)\right) \in L^{p}(0,1 ; X),
$$

and we get $F_{\omega}(f)+\widetilde{R}_{\omega}+S_{\omega} \in L^{p}(0,1 ; X)$. So, by Propositions 4,5 and 6 , statement 2 is satisfied.

\subsection{Statement 2 Implies Statement 1}

Assume statement 2. We have to prove that $u$, defined by (39), is the classical solution of $(3),(4)$. Set

$$
v(x)=\left(I+R_{\omega}\right)^{-1}\left(F_{\omega}(f)(x)+\widetilde{R}_{\omega}(x)+S_{\omega}(x)\right)=\left(L_{\omega}+M_{\omega}\right)^{2} u(x) .
$$

- First step: $u \in L^{p}\left(0,1 ; D\left(\left(L_{\omega}-M_{\omega}\right)^{2}\right)\right)$. From Proposition $4-7$, we get that $v \in L^{p}(0,1 ; X)$. Since

$$
\left(L_{\omega}-M_{\omega}\right)^{2}\left(L_{\omega}+M_{\omega}\right)^{-2} \in \mathcal{L}(X)
$$

we have

$$
x \mapsto\left(L_{\omega}-M_{\omega}\right)^{2} u(x)=\left(L_{\omega}-M_{\omega}\right)^{2}\left(L_{\omega}+M_{\omega}\right)^{-2} v(x) \in L^{p}(0,1 ; X) .
$$

- Second step: $\left(L_{\omega}-M_{\omega}\right) u^{\prime} \in L^{p}(0,1 ; X)$.

Since $v=F_{\omega}(f)+\Gamma_{\omega}-R_{\omega}(v)$, we have

$$
u(.):=\left(L_{\omega}+M_{\omega}\right)^{-2}\left(F_{\omega}(f)(.)+\Gamma_{\omega}(.)\right)-\left(L_{\omega}+M_{\omega}\right)^{-2} R_{\omega}(v)(.),
$$


so, using (32), (33) and (34), we can write $u$ in the following form $u=\bar{u}+\widetilde{u}+\widehat{u}$ where

$$
\left\{\begin{aligned}
\bar{u}(x)= & \left(L_{\omega}+M_{\omega}\right)^{-1} G(f)(x)+\left(L_{\omega}+M_{\omega}\right)^{-1} K(f)(x) \\
& -\frac{1}{2}\left(L_{\omega}+M_{\omega}\right)^{-1} G\left(C_{L_{\omega}, M_{\omega}} v(.)\right)(x)+\frac{1}{2}\left(L_{\omega}+M_{\omega}\right)^{-1} K\left(C_{L_{\omega}, M_{\omega}} v(.)\right)(x), \\
\widetilde{u}(x)= & \left(L_{\omega}+M_{\omega}\right)^{-1} e^{x M_{\omega}} f_{0}, \\
\widehat{u}(x)= & \left(L_{\omega}+M_{\omega}\right)^{-1} e^{(1-x) L_{\omega}} f_{1},
\end{aligned}\right.
$$

with

$$
\begin{aligned}
f_{0}= & -\left(L_{\omega}+H\right) \Lambda_{\omega}^{-1} e^{L_{\omega}} G(f)(1)+\left(L_{\omega}+H\right) \Lambda_{\omega}^{-1} K(f)(0) \\
& +\left(L_{\omega}+H\right) \Lambda_{\omega}^{-1}\left[\left(I-e^{L_{\omega}} e^{M_{\omega}}\right) d_{0}+e^{L_{\omega}}\left(L_{\omega}+M_{\omega}\right) u_{1}\right]+d_{0} \\
& +\frac{1}{2}\left(L_{\omega}+H\right) \Lambda_{\omega}^{-1} K\left(C_{L_{\omega}, M_{\omega}} v(.)\right)(0)+\frac{1}{2}\left(L_{\omega}+H\right) \Lambda_{\omega}^{-1} e^{L_{\omega}} G\left(C_{L_{\omega}, M_{\omega}} v(.)\right)(1),
\end{aligned}
$$

and

$$
\begin{aligned}
f_{1}= & -e^{M_{\omega}}\left(L_{\omega}+H\right) \Lambda_{\omega}^{-1} K(f)(0)+\left(-e^{M_{\omega}} d_{0}+\left(M_{\omega}+L_{\omega}\right) u_{1}\right) \\
& -\left[I-e^{M_{\omega}}\left(L_{\omega}+H\right) \Lambda_{\omega}^{-1} e^{L_{\omega}}\right] G(f)(1) \\
& -e^{M_{\omega}}\left(L_{\omega}+H\right) \Lambda_{\omega}^{-1}\left[\left(I-e^{L_{\omega}} e^{M_{\omega}}\right) d_{0}+e^{L_{\omega}}\left(M_{\omega}+L_{\omega}\right) u_{1}\right] \\
& +\frac{1}{2}\left[I-e^{M_{\omega}}\left(L_{\omega}+H\right) \Lambda_{\omega}^{-1} e^{L_{\omega}}\right] G\left(C_{L_{\omega}, M_{\omega}} v(.)\right)(1) \\
& -\frac{1}{2} e^{M_{\omega}}\left(L_{\omega}+H\right) \Lambda_{\omega}^{-1} K\left(C_{L_{\omega}, M_{\omega}} v(.)\right)(0)
\end{aligned}
$$

We have

$$
\begin{aligned}
\left(L_{\omega}+M_{\omega}\right) \bar{u}^{\prime}(x) & =M_{\omega} G(f)(x)-L_{\omega} K(f)(x)-\frac{1}{2} M_{\omega} G\left(C_{L_{\omega}, M_{\omega}} v(.)\right)(x) \\
& -\frac{1}{2} L_{\omega} K\left(C_{L_{\omega}, M_{\omega}} v(.)\right)(x)-C_{L_{\omega}, M_{\omega}} v(x)
\end{aligned}
$$

but $f \in L^{p}(0,1 ; X)$ and also $v$ (see first step) so, in virtue of Proposition 2 and Lemma 2 , statement 5 , we obtain $\left(L_{\omega}+M_{\omega}\right) \bar{u}^{\prime}(.) \in L^{p}(0,1 ; X)$. Since

$$
\left(L_{\omega}-M_{\omega}\right) \bar{u}^{\prime}(.)=\left(L_{\omega}-M_{\omega}\right)\left(L_{\omega}+M_{\omega}\right)^{-1}\left(L_{\omega}+M_{\omega}\right) \bar{u}^{\prime}(.),
$$

we get

$$
x \mapsto\left(L_{\omega}-M_{\omega}\right) \bar{u}^{\prime}(x) \in L^{p}(0,1 ; X) .
$$

Now in $f_{1}$, putting together the terms containing $e^{M_{\omega}}$; we write

$$
f_{1}=\left(M_{\omega}+L_{\omega}\right) u_{1}-G(f)(1)+\frac{1}{2} G\left(C_{L_{\omega}, M_{\omega}} v(.)\right)(1)+e^{M_{\omega}} \mu_{1},
$$

where $\mu_{1} \in X$. So, from (20) and (36), we deduce that

$$
f_{1} \in\left(D\left(M_{\omega}\right), X\right)_{\frac{1}{p}, p}
$$


and thus

$$
x \mapsto\left(L_{\omega}-M_{\omega}\right) \widehat{u}^{\prime}(x) \in L^{p}(0,1 ; X) .
$$

It remains to study $\widetilde{u}$. For $f_{0}$, we use Lemma 3 , statement 5 ; then, we can write

$$
\begin{aligned}
f_{0} & =\left(L_{\omega}+M_{\omega}\right) \Lambda_{\omega}^{-1} w_{0}+e^{L_{\omega}} e^{M_{\omega}}\left(L_{\omega}+H\right) \Lambda_{\omega}^{-1}\left(w_{0}+d_{0}\right)+e^{L_{\omega}} e^{M_{\omega}} d_{0} \\
& -e^{L_{\omega}}\left(L_{\omega}+M_{\omega}\right) u_{1}+e^{L_{\omega}} G(f)(1)-\frac{1}{2} e^{L_{\omega}} G\left(C_{L_{\omega}, M_{\omega}} v(.)\right)(1) \\
& -K(f)(0)-\frac{1}{2} K\left(C_{L_{\omega}, M_{\omega}} v(.)\right)(0)+\left(L_{\omega}+M_{\omega}\right) \Lambda_{\omega}^{-1} d_{0}
\end{aligned}
$$

where

$$
\begin{aligned}
w_{0}= & -e^{L_{\omega}} e^{M_{\omega}} d_{0}+e^{L_{\omega}}\left(L_{\omega}+M_{\omega}\right) u_{1}-e^{L_{\omega}} G(f)(1) \\
& +K(f)(0)+\frac{1}{2} K\left(C_{L_{\omega}, M_{\omega}} v(.)\right)(0)+\frac{1}{2} e^{L_{\omega}} G\left(C_{L_{\omega}, M_{\omega}} v(.)\right)(1) .
\end{aligned}
$$

So

$$
f_{0}=\left(L_{\omega}+M_{\omega}\right) \Lambda_{\omega}^{-1} w_{0}-K(f)(0)-\frac{1}{2} K\left(C_{L_{\omega}, M_{\omega}} v(.)\right)(0)+\left(L_{\omega}+M_{\omega}\right) \Lambda_{\omega}^{-1} d_{0}+e^{L_{\omega}} \mu_{0},
$$

where $\mu_{0} \in X$. It's easily seen that $w_{0} \in\left(D\left(M_{\omega}\right), X\right)_{\frac{1}{p}, p}$ so that

$$
f_{0} \in\left(D\left(M_{\omega}\right), X\right)_{\frac{1}{p}, p}
$$

and

$$
x \mapsto\left(L_{\omega}-M_{\omega}\right) \widetilde{u}^{\prime}(x) \in L^{p}(0,1 ; X) .
$$

Finally, (41), (42) and (43) prove that $x \mapsto\left(L_{\omega}-M_{\omega}\right) u^{\prime}(x) \in L^{p}(0,1 ; X)$.

- Third step: $u$ satisfies (3). From this step and the others, we will deduce that $u \in W^{2, p}(0,1 ; X)$ since

$$
x \mapsto u^{\prime \prime}(x)=-\left(L_{\omega}-M_{\omega}\right) u^{\prime}(x)+\frac{1}{2}\left(L_{\omega} M_{\omega}+M_{\omega} L_{\omega}\right) u(x)+f(x) \in L^{p}(0,1 ; X) .
$$

Moreover, we have for almost all $x \in(0,1)$

$$
\begin{aligned}
v(x) & =\left(L_{\omega}+M_{\omega}\right) G(f)(x)+\left(L_{\omega}+M_{\omega}\right) K(f)(x) \\
& -\frac{1}{2}\left(L_{\omega}+M_{\omega}\right) G\left(C_{L_{\omega}, M_{\omega}} v(.)\right)(x)+\frac{1}{2}\left(L_{\omega}+M_{\omega}\right) K\left(C_{L_{\omega}, M_{\omega}} v(.)\right)(x) \\
& +\left(L_{\omega}+M_{\omega}\right) e^{x M_{\omega}} f_{0}+\left(L_{\omega}+M_{\omega}\right) e^{(1-x) L_{\omega}} f_{1},
\end{aligned}
$$

and

$$
\begin{aligned}
& \left(L_{\omega}+M_{\omega}\right) u^{\prime}(x)=M_{\omega} G(f)(x)-L_{\omega} K(f)(x)-\frac{1}{2} M_{\omega} G\left(C_{L_{\omega}, M_{\omega}} v(.)\right)(x) \\
& -\frac{1}{2} L_{\omega} K\left(C_{L_{\omega}, M_{\omega}} v(.)\right)(x)+M_{\omega} e^{x M_{\omega}} f_{0}-L_{\omega} e^{(1-x) L_{\omega}} f_{1}-C_{L_{\omega}, M_{\omega}} v(x) .
\end{aligned}
$$


Therefore, inserting (44) in $\left(L_{\omega}+M_{\omega}\right) u^{\prime}(x)$, and using Lemma 3 , statement 6 , we have for almost all $x \in(0,1)$

$$
\begin{aligned}
u^{\prime}(x) & =M_{\omega}\left(L_{\omega}+M_{\omega}\right)^{-1} G(f)(x)-L_{\omega}\left(L_{\omega}+M_{\omega}\right)^{-1} K(f)(x) \\
& -\frac{1}{2} M_{\omega}\left(L_{\omega}+M_{\omega}\right)^{-1} G\left(C_{L_{\omega}, M_{\omega}} v(.)\right)(x)-\frac{1}{2} L_{\omega}\left(L_{\omega}+M_{\omega}\right)^{-1} K\left(C_{L_{\omega}, M_{\omega}} v(.)\right)(x) \\
& +M_{\omega}\left(L_{\omega}+M_{\omega}\right)^{-1} e^{x M_{\omega}} f_{0}-L_{\omega}\left(L_{\omega}+M_{\omega}\right)^{-1} e^{(1-x) L_{\omega}} f_{1} .
\end{aligned}
$$

Then for almost all $x \in(0,1)$

$$
\begin{aligned}
u^{\prime \prime}(x) & =f(x)+M_{\omega}\left(L_{\omega}+M_{\omega}\right)^{-1} M_{\omega} G(f)(x)+L_{\omega}\left(L_{\omega}+M_{\omega}\right)^{-1} L_{\omega} K(f)(x) \\
& -\frac{1}{2} M_{\omega}\left(L_{\omega}+M_{\omega}\right)^{-1} M_{\omega} G\left(C_{L_{\omega}, M_{\omega}} v(.)\right)(x)+\frac{1}{2} L_{\omega}\left(L_{\omega}+M_{\omega}\right)^{-1} L_{\omega} K\left(C_{L_{\omega}, M_{\omega}} v(.)\right)(x) \\
& +\frac{1}{2}\left(L_{\omega}-M_{\omega}\right)\left(L_{\omega}+M_{\omega}\right)^{-1} C_{L_{\omega}, M_{\omega}} v(x) \\
& +M_{\omega}\left(L_{\omega}+M_{\omega}\right)^{-1} M_{\omega} e^{x M_{\omega}} f_{0}+L_{\omega}\left(L_{\omega}+M_{\omega}\right)^{-1} L_{\omega} e^{(1-x) L_{\omega}} f_{1},
\end{aligned}
$$

we insert (44) in (46), so we obtain for almost all $x \in(0,1)$

$$
\begin{aligned}
u^{\prime \prime}(x)= & f(x)+E_{1} G(f)(x)+T_{1} K(f)(x)-\frac{1}{2} E_{1} G\left(C_{L_{\omega}, M_{\omega}} v\right)(x) \\
& +\frac{1}{2} T_{1} K\left(C_{L_{\omega}, M_{\omega}} v\right)(x)+E_{1} e^{x M} f_{0}+T_{1} e^{(1-x) L} f_{1},
\end{aligned}
$$

where, on the domain $D\left(L_{\omega}+M_{\omega}\right)$

$$
\begin{aligned}
E_{1} & =M_{\omega}\left(L_{\omega}+M_{\omega}\right)^{-1} M_{\omega}+\frac{1}{2}\left(L_{\omega}-M_{\omega}\right)\left(L_{\omega}+M_{\omega}\right)^{-1} C_{L_{\omega}, M_{\omega}}\left(L_{\omega}+M_{\omega}\right) \\
& =\frac{1}{2} M_{\omega}-\frac{1}{2}\left(L_{\omega}-M_{\omega}\right) M_{\omega}\left(L_{\omega}+M_{\omega}\right)^{-1}
\end{aligned}
$$

and

$$
\begin{aligned}
T_{1} & =L_{\omega}\left(L_{\omega}+M_{\omega}\right)^{-1} L_{\omega}+\frac{1}{2}\left(L_{\omega}-M_{\omega}\right)\left(L_{\omega}+M_{\omega}\right)^{-1} C_{L_{\omega}, M_{\omega}}\left(L_{\omega}+M_{\omega}\right) \\
& =\frac{1}{2} L_{\omega}+\frac{1}{2}\left(L_{\omega}-M_{\omega}\right) L_{\omega}\left(L_{\omega}+M_{\omega}\right)^{-1},
\end{aligned}
$$

in virtue of Lemma 3, statement 6. Using (40), (45) and (47), we have for almost all $x \in(0,1)$

$$
\begin{aligned}
& u^{\prime \prime}(x)+\left(L_{\omega}-M_{\omega}\right) u^{\prime}(x)-\frac{1}{2}\left(L_{\omega} M_{\omega}+M_{\omega} L_{\omega}\right) u(x) f(x)+E_{2} G(f)(x)+T_{2} K(f)(x) \\
& -\frac{1}{2} E_{2} G\left(C_{L_{\omega}, M_{\omega}} v(.)\right)(x)+\frac{1}{2} T_{2} K\left(C_{L_{\omega}, M_{\omega}} v(.)\right)(x)+E_{2} e^{x M_{\omega}} f_{0}+T_{2} e^{(1-x) L_{\omega}} f_{1},
\end{aligned}
$$

where

$$
\begin{aligned}
E_{2}= & \frac{1}{2} M_{\omega}-\frac{1}{2}\left(L_{\omega}-M_{\omega}\right) M_{\omega}\left(L_{\omega}+M_{\omega}\right)^{-1}+\left(L_{\omega}-M_{\omega}\right) M_{\omega}\left(L_{\omega}+M_{\omega}\right)^{-1} \\
& -\frac{1}{2}\left(L_{\omega} M_{\omega}+M_{\omega} L_{\omega}\right)\left(L_{\omega}+M_{\omega}\right)^{-1},
\end{aligned}
$$


and

$$
\begin{aligned}
T_{2}= & \frac{1}{2} L_{\omega}+\frac{1}{2}\left(L_{\omega}-M_{\omega}\right) L_{\omega}\left(L_{\omega}+M_{\omega}\right)^{-1}-\left(L_{\omega}-M_{\omega}\right) L_{\omega}\left(L_{\omega}+M_{\omega}\right)^{-1} \\
& -\frac{1}{2}\left(L_{\omega} M_{\omega}+M_{\omega} L_{\omega}\right)\left(L_{\omega}+M_{\omega}\right)^{-1} .
\end{aligned}
$$

But in virtue of Lemma 3, statement 8, we obtain

$$
E_{2} G(f)(.)=T_{2} K(f)(.)=0 \text { in } L^{p}(0,1 ; X) .
$$

We finally get

$$
u^{\prime \prime}(x)+\left(L_{\omega}-M_{\omega}\right) u^{\prime}(x)-\frac{1}{2}\left(L_{\omega} M_{\omega}+M_{\omega} L_{\omega}\right) u(x)=f(x) .
$$

We conclude that $u$, determined by (39), is the classical solution of (3), (4).

\section{Go Back to the Commutative Case}

This section is devoted to some comparison with the recent paper [1]. We will show that this work improves the results contained in [1]. In fact, instead of considering families $\left(L_{\omega}\right)_{\omega \geqslant \omega_{0}},\left(M_{\omega}\right)_{\omega \geqslant \omega_{0}}$ of linear operators, we consider $L, M$ two closed linear operators in $X$ such that

$$
\left\{\begin{array}{l}
D(M)=D(L) \\
L M=M L
\end{array}\right.
$$

as assumptions (11) and (12) in [1, p. 59]. But we do not assume the commutativities between $L$ and $H$ and between $M$ and $H$, as (15) and (16) in [1, p. 59].

Note that here, in virtue of (48), we have

$$
\Lambda=(M-H)+e^{L} e^{M}(L+H)=(M-H)+e^{L+M}(L+H),
$$

and since $C_{L, M}=0$ we have $R=0$. Then, by formula (21), the solution of Problem

$$
\left\{\begin{array}{l}
u^{\prime \prime}(x)+(L-M) u^{\prime}(x)-L M u(x)=f(x), x \in(0,1), \\
u^{\prime}(0)-H u(0)=d_{0}, u(1)=u_{1},
\end{array}\right.
$$

is

$$
u(.)=(L+M)^{-2}(F(f)(.)+\Gamma(.)),
$$

(here $\left.(I+R)^{-1}=I\right)$. Moreover, for $x \in[0,1]$

$$
\begin{aligned}
& (L+M)^{-2} F(f)(x) \\
= & (L+M)^{-1} \int_{0}^{x} e^{(x-s) M} f(s) d s+(L+M)^{-1} \int_{x}^{1} e^{(s-x) L} f(s) d s \\
& -(L+M)^{-1} e^{x M}(L+H) \Lambda^{-1} e^{L} \int_{0}^{1} e^{(1-s) M} f(s) d s \\
& +(L+M)^{-1} e^{x M}(L+H) \Lambda^{-1} \int_{0}^{1} e^{s L} f(s) d s \\
& -(L+M)^{-1} e^{(1-x) L} e^{M}(L+H) \Lambda^{-1} \int_{0}^{1} e^{s L} f(s) d s \\
& -(L+M)^{-1} e^{(1-x) L}\left[I-e^{M}(L+H) \Lambda^{-1} e^{L}\right] \int_{0}^{1} e^{(1-s) M} f(s) d s,
\end{aligned}
$$


and

$$
\begin{aligned}
& (L+M)^{-2} \Gamma(x) \\
= & (L+M)^{-1} e^{x M}\left[(L+H) \Lambda^{-1}+I-(L+H) \Lambda^{-1} e^{L+M}\right] d_{0} \\
& -(L+M) e^{(1-x) L} e^{M}\left[(L+H) \Lambda^{-1}+I-(L+H) \Lambda^{-1} e^{L+M}\right] d_{0} \\
& +(L+M)^{-1} e^{x M}(L+H) \Lambda^{-1} e^{L}(L+M) u_{1} \\
& +(L+M)^{-1} e^{(1-x) L}\left[I-e^{M}(L+H) \Lambda^{-1} e^{L}\right](L+M) u_{1} .
\end{aligned}
$$

Now, using the fact that

$$
(L+H) \Lambda^{-1}=(L+M) \Lambda^{-1}+e^{L+M}(L+H) \Lambda^{-1}-I,
$$

(see Lemma 3, statement 5), we deduce

$$
\begin{aligned}
& e^{M} d_{0}+e^{M}(L+H) \Lambda^{-1} d_{0}-e^{M}(L+H) \Lambda^{-1} e^{L+M} d_{0} \\
= & e^{M} d_{0}+e^{M}\left[(L+M) \Lambda^{-1}+e^{L+M}(L+H) \Lambda^{-1}-I\right] d_{0} \\
& -e^{M}(L+H) \Lambda^{-1} e^{L+M} d_{0} \\
= & e^{M}(L+M) \Lambda^{-1} d_{0}+e^{M} e^{L+M}(L+H) \Lambda^{-1} d_{0} \\
& -e^{M}(L+H) \Lambda^{-1} e^{L+M} d_{0} \\
= & e^{M}(L+M) \Lambda^{-1} d_{0}+e^{M}\left[e^{L+M} ;(L+H) \Lambda^{-1}\right] d_{0},
\end{aligned}
$$

and since

$$
\begin{aligned}
& (L+H) \Lambda^{-1} d_{0}+d_{0}-(L+H) \Lambda^{-1} e^{L+M} d_{0} \\
= & {\left[(L+H)+(M-H)+e^{L+M}(L+H)\right] \Lambda^{-1} d_{0}-(L+H) \Lambda^{-1} e^{L+M} d_{0} } \\
= & {\left[(L+M)+e^{L+M}(L+H)\right] \Lambda^{-1} d_{0}-(L+H) \Lambda^{-1} e^{L+M} d_{0} } \\
= & (L+M) \Lambda^{-1} d_{0}+\left[e^{L+M} ;(L+H) \Lambda^{-1}\right] d_{0},
\end{aligned}
$$

then we obtain

$$
\begin{aligned}
& (L+M)^{-2} \Gamma(x) \\
= & e^{x M} \Lambda^{-1} d_{0}+(L+M)^{-1} e^{x M}\left[e^{L+M} ;(L+H) \Lambda^{-1}\right] d_{0} \\
& -e^{(1-x) L} e^{M} \Lambda^{-1} d_{0}-(L+M)^{-1} e^{(1-x) L} e^{M}\left[e^{L+M} ;(L+H) \Lambda^{-1}\right] d_{0} \\
& +(L+M)^{-1} e^{x M}(L+H) \Lambda^{-1} e^{L}(L+M) u_{1} \\
& +(L+M)^{-1} e^{(1-x) L}\left[I-e^{M}(L+H) \Lambda^{-1} e^{L}\right](L+M) u_{1} .
\end{aligned}
$$


Finally, the representation solution of Problem (49), for a.e $x \in(0,1)$, is

$$
\begin{aligned}
& u(x) \\
= & e^{x M} \Lambda^{-1} d_{0}+(L+M)^{-1} e^{x M}(L+H) \Lambda^{-1} e^{L}(L+M) u_{1} \\
& -(L+M)^{-1} e^{x M}(L+H) \Lambda^{-1} e^{L} \int_{0}^{1} e^{(1-s) M} f(s) d s \\
& +(L+M)^{-1} e^{x M}(L+H) \Lambda^{-1} \int_{0}^{1} e^{s L} f(s) d s \\
& -e^{(1-x) L} e^{M} \Lambda^{-1} d_{0}+(L+M)^{-1} e^{(1-x) L}\left[I-e^{M}(L+H) \Lambda^{-1} e^{L}\right](L+M) u_{1} \\
& -(L+M)^{-1} e^{(1-x) L} e^{M}(L+H) \Lambda^{-1} \int_{0}^{1} e^{s L} f(s) d s \\
& -(L+M)^{-1} e^{(1-x) L}\left[I-e^{M}(L+H) \Lambda^{-1} e^{L}\right] \int_{0}^{1} e^{(1-s) M} f(s) d s \\
& +(L+M)^{-1} \int_{0}^{x} e^{(x-s) M} f(s) d s+(L+M)^{-1} \int_{x}^{1} e^{(s-x) L} f(s) d s \\
& +(L+M)^{-1}\left(e^{x M}-e^{(1-x) L} e^{M}\right)\left[e^{L+M} ;(L+H) \Lambda^{-1}\right] d_{0} .
\end{aligned}
$$

This representation generalizes the one used in [1, p. 63]. Note that, the last term

$$
(L+M)^{-1}\left(e^{x M}-e^{(1-x) L} e^{M}\right)\left[e^{L+M} ;(L+H) \Lambda^{-1}\right] d_{0},
$$

vanishes when $H$ and operators $L$ and $M$ commute in the sense of the resolvent operators and then, our solution coincides with the one used in [1].

Corollary 1. Let $L, M$ be closed linear operators in $X$ satisfying (48). Assume (6) (14) where $L_{\omega}=L, M_{\omega}=M$ for any $\omega \geqslant \omega_{0}$. Let $f \in L^{p}(0,1 ; X)$ with $1<p<\infty$. Then, the two following assertions are equivalent

1. Problem (49) has a unique classical solution.

2. $u_{1}, \Lambda^{-1} d_{0} \in\left(X, D\left((L+M)^{2}\right)\right)_{1-\frac{1}{2 p}, p}$.

Remark 6. Note that, instead of commutativity between $H$ and operators $L$ and $M$, we have assumed the following hypothesis

$$
\forall \xi \in D(L)=D(M)=D(L+M), \Lambda^{-1} \xi \in D\left((L+M)^{2}\right),
$$

which is obviously verified when the operator $H$ commutes with $L$ and $M$ in the sense of the resolvent operators. Notice also that (48) implies (10).

\section{Application}

Let $X=L^{2}(\mathbb{R})$. Define operators $L_{\omega}, M_{\omega}$ and $H$ by

$$
\left\{\begin{array}{l}
D\left(L_{\omega}\right)=D\left(M_{\omega}\right)=H^{2}(\mathbb{R}), \quad D(H)=H^{1}(\mathbb{R}), \\
L_{\omega} \varphi(y)=\varphi^{\prime \prime}(y)+a(y) \varphi^{\prime}(y)-\omega^{\alpha} \varphi(y), \quad M_{\omega} \varphi(y)=\varphi^{\prime \prime}(y)-\omega^{\alpha} \varphi(y), \\
H \varphi(y)=\varphi^{\prime}(y),
\end{array}\right.
$$


where $\alpha>0, \omega>0$ and $a \in C_{b}^{2}(\mathbb{R}), a \neq 0$. It is not difficult to prove that $L_{\omega}, M_{\omega}$ generate analytic semigroups and that

$$
L_{\omega}+M_{\omega} \text { is boundedly invertible. }
$$

On the other hand, we have the following properties:

$$
\begin{aligned}
& \left\{\begin{array}{l}
D\left(\left(L_{\omega}+M_{\omega}\right)^{2}\right)=H^{4}(\mathbb{R}) \subset D\left(\left(L_{\omega}-M_{\omega}\right)^{2}\right)=H^{3}(\mathbb{R}) \\
D\left(M_{\omega} L_{\omega}\right)=D\left(L_{\omega} M_{\omega}\right)=H^{4}(\mathbb{R})
\end{array}\right. \\
& \left(M_{\omega} L_{\omega}\right) \varphi(y)=\varphi^{(4)}(y)+a(y) \varphi^{(3)}(y)+2\left(a^{\prime}(y)-\omega^{\alpha}\right) \varphi^{\prime \prime}(y) \\
& +\left(a^{\prime \prime}(y)-\omega^{\alpha} a(y)\right) \varphi^{\prime}(y)+\omega^{2 \alpha} \varphi(y) \\
& \left(L_{\omega} M_{\omega}\right) \varphi(y)=\varphi^{(4)}(y)+a(y) \varphi^{(3)}(y)-2 \omega^{\alpha} \varphi^{\prime \prime}(y)-\omega^{\alpha} \varphi^{\prime}(y)+\omega^{2 \alpha} \varphi(y) ;
\end{aligned}
$$

note that $L_{\omega} M_{\omega} \neq M_{\omega} L_{\omega}$. We have

$$
\left\{\begin{array}{l}
D\left(M_{\omega}-H\right)=H^{2}(\mathbb{R}), \\
\left(M_{\omega}-H\right) \varphi(y)=\varphi^{\prime \prime}(y)-\omega^{\alpha} \varphi(y)-\varphi^{\prime}(y)=\psi(y) .
\end{array}\right.
$$

From the Fourier transform, $M_{\omega}-H$ is invertible and

$$
\left[\left(M_{\omega}-H\right)^{-1} \psi\right](y)=-\int_{\mathbb{R}} \frac{e^{2 i \pi y \xi} \widehat{\psi}(\xi)}{\left(\omega^{\alpha}+4 \pi^{2} \xi^{2}+2 i \pi \xi\right)} d \xi .
$$

So

$$
\begin{aligned}
\Lambda_{\omega} & =\left(M_{\omega}-H\right)+e^{L_{\omega}} e^{M_{\omega}}\left(L_{\omega}+H\right) \\
& =\left(I+e^{L_{\omega}} e^{M_{\omega}}\left(L_{\omega}+H\right)\left(M_{\omega}-H\right)^{-1}\right)\left(M_{\omega}-H\right),
\end{aligned}
$$

and

$$
\begin{aligned}
& \left(L_{\omega}+H\right)\left[\left(M_{\omega}-H\right)^{-1} \psi\right](y) \\
& =L_{\omega}\left[\left(M_{\omega}-H\right)^{-1} \psi\right](y)+H\left[\left(M_{\omega}-H\right)^{-1} \psi\right](y) \\
& =\int_{\mathbb{R}} \frac{4 \pi^{2} \xi^{2} e^{2 i \pi y \xi} \widehat{\psi}(\xi)}{\left(\omega^{\alpha}+4 \pi^{2} \xi^{2}+2 i \pi \xi\right)} d \xi-(a(y)+1) \int_{\mathbb{R}} \frac{2 i \pi \xi e^{2 i \pi y \xi} \widehat{\psi}(\xi)}{\left(\omega^{\alpha}+4 \pi^{2} \xi^{2}+2 i \pi \xi\right)} d \xi \\
& +\omega^{\alpha} \int_{\mathbb{R}} \frac{e^{2 i \pi y \xi} \widehat{\psi}(\xi)}{\left(\omega^{\alpha}+4 \pi^{2} \xi^{2}+2 i \pi \xi\right)} d \xi
\end{aligned}
$$

then

$$
\begin{aligned}
\left\|\left(L_{\omega}+H\right)\left[\left(M_{\omega}-H\right)^{-1} \psi\right]\right\|_{L^{2}(\mathbb{R})} & \leqslant C\|\widehat{\psi}\|_{L^{2}(\mathbb{R})}+C \omega^{\alpha}\|\widehat{\psi}\|_{L^{2}(\mathbb{R})} \\
& =C\left(1+\omega^{\alpha}\right)\|\psi\|_{L^{2}(\mathbb{R})}
\end{aligned}
$$

and

$$
\begin{aligned}
\left\|e^{L_{\omega}} e^{M_{\omega}}\left(L_{\omega}+H\right)\left(M_{\omega}-H\right)^{-1} \psi\right\| & \leq C\left(1+\omega^{\alpha}\right)\left\|e^{L_{\omega}}\right\|\left\|e^{M_{\omega}}\right\|\|\psi\|_{L^{2}(\mathbb{R})} \\
& \leq \frac{C}{\omega^{2 \alpha}}\left(1+\omega^{\alpha}\right)\|\psi\|_{L^{2}(\mathbb{R})} .
\end{aligned}
$$


Therefore, for a large $\omega$, we deduce that $\Lambda_{\omega}$ is boundedly invertible.

Now, notice that

$$
\forall \varphi \in D\left(L_{\omega}\right)=H^{2}(\mathbb{R}), \Lambda_{\omega}^{-1} \varphi \in D\left(\left(L_{\omega}+M\right)^{2}\right)=H^{4}(\mathbb{R}) .
$$

Set $\Lambda_{\omega}^{-1} \varphi=\psi$, then

$$
\varphi=\Lambda_{\omega} \psi=\left[(M-H)+e^{L_{\omega}} e^{M}\left(L_{\omega}+H\right)\right] \psi,
$$

thus

$$
\left(M_{\omega}-H\right) \psi=\psi^{\prime \prime}-\omega^{\beta} \psi-\psi^{\prime} \in D\left(L_{\omega}\right)=H^{2}(\mathbb{R})
$$

which implies

$$
\Lambda_{\omega}^{-1} \varphi=\psi \in H^{4}(\mathbb{R}) .
$$

Moreover, there exists $C>0$ such that, for any $\omega>0$,

$$
\left\|\left[M_{\omega}, L_{\omega}\right]\left(L_{\omega}+M_{\omega}\right)^{-2}\right\|_{L(X)} \leq \frac{C}{\omega \gamma},
$$

where $\gamma=2 \alpha$ if $0<\omega<1$ and $\gamma=\alpha$ if $\omega \geqslant 1$.

Therefore, all our results apply for a large $\omega>0$, to the following boundary value problem:

$$
\left\{\begin{array}{l}
\frac{\partial^{2} u}{\partial x^{2}}(x, y)+2 \frac{\partial^{3} u}{\partial y^{2} \partial x}(x, y)+a(y) \frac{\partial^{2} u}{\partial y \partial x}(x, y)-\frac{\partial^{4} u}{\partial y^{4}}(x, y) \\
-a(y) \frac{\partial^{3} u}{\partial y^{3}}(x, y)-a^{\prime}(y) \frac{\partial^{2} u}{\partial y^{2}}(x, y)-\frac{1}{2} a^{\prime \prime}(y) \frac{\partial u}{\partial y}(x, y)+\omega^{\alpha} a(y) \frac{\partial u}{\partial y}(x, y) \\
+\omega^{\alpha} \frac{\partial u}{\partial y}(x, y)+2 \omega^{\alpha} \frac{\partial^{2} u}{\partial y^{2}}(x, y)-\omega^{2 \alpha} u(x, y)=f(x, y), x \in(0,1), y \in \mathbb{R}, \\
\frac{\partial u}{\partial x}(0, y)-\frac{\partial u}{\partial y} u(0, y)=d_{0}, y \in \mathbb{R}, \\
u(1, y)=u_{1}, y \in \mathbb{R} .
\end{array}\right.
$$

Proposition 8. Let $f \in L^{p}\left(0,1 ; L^{2}(\mathbb{R})\right)$ with $1<p<+\infty$. Then, for a large $\omega>0$, the two following assertions are equivalent.

1. Problem (50) has a classical solution $u$.

2. $u_{1}, \Lambda_{\omega}^{-1} d_{0} \in\left(H^{4}(\mathbb{R}), L^{2}(\mathbb{R})\right)_{\frac{1}{2 p}, p}$,

where $\left(H^{4}(\mathbb{R}), L^{2}(\mathbb{R})\right)_{\frac{1}{2 p}, p}=B_{2, p}^{4(1-1 / 2 p)}(\mathbb{R})$ (see [6, p. 680-681]).

Let us explicit $\Lambda_{\omega}^{-1} d_{0}$. Setting $V_{\omega}=e^{L_{\omega}} e^{M_{\omega}}\left(L_{\omega}+H\right)\left(M_{\omega}-H\right)^{-1}$ and noting that

$$
\left(I+V_{\omega}\right)^{-1}=I-V_{\omega}\left(I+V_{\omega}\right)^{-1} \text {, }
$$

we have

$$
\begin{aligned}
\Lambda_{\omega}^{-1} d_{0} & =\left(M_{\omega}-H\right)^{-1}\left(I+V_{\omega}\right)^{-1} d_{0} \\
& =\left(M_{\omega}-H\right)^{-1} d_{0}-\left(M_{\omega}-H\right)^{-1} V_{\omega}\left(I+V_{\omega}\right)^{-1} d_{0} .
\end{aligned}
$$

But $V_{\omega}$ contains the regularizing operator $e^{L_{\omega}}$, then $\Lambda_{\omega}^{-1} d_{0} \in B_{2, p}^{4(1-1 / 2 p)}(\mathbb{R})$ if and only if $\left(M_{\omega}-H\right)^{-1} d_{0} \in B_{2, p}^{4(1-1 / 2 p)}(\mathbb{R})$, that is

$$
y \rightarrow \int_{\mathbb{R}} \frac{e^{2 i \pi y \xi} \widehat{d}_{0}(\xi)}{\left(\omega^{\alpha}+4 \pi^{2} \xi^{2}+2 i \pi \xi\right)} d \xi \in B_{2, p}^{4(1-1 / 2 p)}(\mathbb{R}) .
$$




\section{References}

1. Cheggag M., Favini A., Labbas R., Maingot S., Medeghri A. Elliptic Problems with Robin Boundary Coefficient-Operator Conditions in General $L_{p}$ Sobolev Spaces and Applications. Bulletin of the South Ural State University. Series: Mathematical Modelling, Programming and Computer Software, 2015, vol. 8, no. 3, pp. 56-77. DOI: 10.14529/mmp150304

2. Favini A., Labbas R., Maingot S., Meisner M. Study of Complete Abstract Elliptic Differential Equations in Non Commutative Cases. Applicable Analysis, 2012, vol. 91, issue 8, pp. 1495-1510. DOI: 10.10801.00036811.2011.635652

3. Favini A., Labbas R., Maingot S., Meisner M. Boundary Value Problem For Elliptic Differential Equations in Non-Commutative Cases. Discrete and Continuous Dynamics System. Series A, 2013, vol. 33, issue 11-12, pp. 4967-4990. DOI: 10.3934/dcds.2013.33.4967

4. Prüss J., Sohr H. On Operators with Bounded Imaginary Powers in Banach Spaces. Mathematische Zeitschrift, 1990, vol. 203, issue 3, pp. 429-452.

5. Cheggag M., Favini A., Labbas R., Maingot S., Medeghri A. Complete Abstract Differential Equations of Elliptic Type with General Robin Boundary Conditions, in UMD Spaces. Discrete and Continuous Dynamics System. Series S, 2011, vol. 4, no. 3, pp. 523-538. DOI: $10.3934 /$ dcdss.2011.4.523

6. Grisvard P. Spazi di tracce ed applicazioni. Rendiconti di matematica. Serie VI, 1972, vol. 5, pp. 657-729. (in Italian)

7. Meisner M. Etude unifiée d'équations aux dérivées partielles de type elliptique régies par des équations différentielles à coefficients opérateurs dans un cadre non commutatif: applications concrètes dans les espaces de Hölder et les espaces $L^{p}$. Thèse de doctorat de l'Université du Havre, 2012.

8. Dore G., Venni A. On the Closedness of the Sum of Two Closed Operators. Mathematische Zeitschrift, 1987, vol. 196, issue 2, pp. 189-201. DOI: 10.1007/BF01163654

9. Favini A., Labbas R., Maingot S., Tanabe H., Yagi A. A Simplified Approach in the Study of Elliptic Differential Equation in UMD Space and New Applications. Funkcialaj Ekvacioj, 2008, vol. 51, no. 2, pp. 165-187.

10. Favini A., Labbas R., Maingot S., Tanabe H., Yagi A. Complete Abstract Differential Equation of Elliptic Type in UMD Spaces. Funkcialaj Ekvacioj, 2006, vol. 49, no. 2, pp. 193-214.

11. Lunardi A., Analytic Semigroups and Optimal Regularity in Parabolic Problems. Basel, Birkhäuser, 1995.

12. Triebel H. Interpolation Theory, Function Spaces, Differential Operators. Amsterdam, N.Y., Oxford, North-Holland, 1978.

13. Dore G. $L^{p}$ Regularity for Abstract Differential Equation. Functional Analysis and Related Topics. Kyoto 1991. Lecture Notes in Mathematics. Vol. 1540. 1993, Berlin, Springer-Verlag, pp. 25-38. DOI: $10.1007 / \mathrm{BFb} 0085472$

Received November 9, 2016 


\title{
НОВЫЕ РЕЗУЛЬТАТЫ О ПОЛНЫХ ЭЛЛИПТИЧЕСКИХ УРАВНЕНИЯХ С КОЭФФИЦИЕНТНО-ОПЕРАТОРНЫМИ ГРАНИЧНЫМИ УСЛОВИЯМИ ТИПА РОБИН В НЕКОММУТАТИВНОМ СЛУЧАЕ
}

\author{
М. Чеггаг, А. Фавини, Р. Лаббас, С. Менго, Х.У. Мелха
}

\begin{abstract}
В статье доказываются некоторые новые результаты для дифференциальных уравнений эллиптического типа второго порядка с общими граничными условиями типа Робина с некоммутативными структурами. Исследование выполнено при условии, что второй член принадлежит пространствам Соболева. Существование, единственность и оптимальная регулярность классического решения доказаны с использованием теории интерполяции, и получены результаты для класса операторов с ограниченными мнимыми степенями. В работе приводится пример применения данной теории. Результаты, полученные в этой работе, улучшают исследования в коммутативном случае М. Чегага, А. Фавини, Р. Лаббаса, С. Менго и А. Медегри. Вводя в рассмотрение некоторые операторные коммутаторы, обобщено представление решения задачи в коммутативном случае и доказано, что это представление обладает свойством регулярности.

Ключевые слова: эллиптическое дифференциальное уравнение второго порядка; граничные условия Робин в случаях коммутативности; аналитические полугруппы; максимальная регулярность.
\end{abstract}

Мустафа Чеггаг, Национальная политехническая школа (г. Оран, Алжир), mustapha.cheggag@enp-oran.dz

Анджело Фавини, кафедра математики, Болонский университет (г. Болонья, Италия), favini@dm.unibo.it.

Раба Лаббас, Нормандский университет; лаборатория прикладной математики, Университет Гавра (г. Гавр, Франция), rabah.labbas@univ-lehavre.fr.

Стефан Менго, Нормандский университет; лаборатория прикладной математики, Университет Гавра (г. Гавр, Франция), stephane.maingot@univ-lehavre.fr.

Хеллаф Ульд Мелха, лаборатория чистой и прикладной математики, Университет имени Абдул Хамид Ибн-Бадиса (г. Мостаганем, Алжир), ould_melha_khel@yahoo.fr.

Поступила в редакцию 9 ноября 2016 г. 